\title{
Lattice results for the longitudinal spin structure and color forces on quarks in a nucleon
}

\author{
S. Bürger, ${ }^{1}$ T. Wurm, ${ }^{1}$ M. Löffler $\odot,{ }^{1}$ M. Göckeler $\odot,{ }^{1}$ G. Bali, ${ }^{1}$ S. Collins, ${ }^{1}$ A. Schäfer, ${ }^{1}$ and A. Sternbeck $\odot^{2}$ \\ (RQCD Collaboration) \\ ${ }^{1}$ University of Regensburg, 93040 Regensburg, Germany \\ ${ }^{2}$ Friedrich Schiller University Jena, Max-Wien-Platz 1, 07743 Jena, Germany
}

(Received 23 November 2021; accepted 15 February 2022; published 8 March 2022)

\begin{abstract}
Using lattice QCD, we calculate the twist-2 contribution $a_{2}$ to the third Mellin moment of the spin structure functions $g_{1}$ and $g_{2}$ in the nucleon. In addition we evaluate the twist-3 contribution $d_{2}$. Our computations make use of $N_{f}=2+1$ gauge field ensembles generated by the Coordinated Lattice Simulations effort. Neglecting quark-line disconnected contributions we obtain as our best estimates $a_{2}^{(p)}=0.069(17), d_{2}^{(p)}=0.0105(68)$ and $a_{2}^{(n)}=0.0068(88), d_{2}^{(n)}=-0.0009(70)$ for the proton and the neutron, respectively, where we use the normalizations given in Eqs. (58) and (59). While the $a_{2}$ results have been converted to the $\overline{\mathrm{MS}}$ scheme using three-loop perturbation theory, the numbers for $d_{2}$ are given in the regularization independent momentum subtraction scheme, i.e., the conversion has been performed only in tree-level perturbation theory. The $d_{2}$ results can be interpreted as corresponding to a transverse color Lorentz force on a quark in a transversely polarized proton of size $F^{(u)}=116(61)$ and $F^{(d)}=$ $-38(66) \mathrm{MeV} / \mathrm{fm}$ for $u$ and $d$ quarks, respectively. The error estimates quoted include statistical and systematic uncertainties added in quadrature.
\end{abstract}

DOI: 10.1103/PhysRevD.105.054504

\section{INTRODUCTION}

For a number of reasons hadron spin structure has attracted intense interest for more than two decades with no sign of attenuation. Quite to the contrary, CEBAF@12 GeV [1] and the EIC [2] promise to bring such investigations to a higher level both with respect to the precision and the variety of observables investigated. This provides a strong motivation also to update theory predictions for the relevant spindependent quantities. The central goal of the JLAB and BNL programs is to better understand the structure of hadrons. This includes multiparton correlations, which are parametrized by higher-twist coefficients.

The most prominent such example is the matrix element $d_{2}$, the third Mellin moment $\left(x^{2}\right)$ of the twist- 3 contribution to the helicity structure function $g_{2}\left(x, Q^{2}\right)$ of deep-inelastic longitudinally polarized lepton-nucleon scattering. As this corresponds to the lowest-dimensional nontrivial chiral-even twist3 matrix element, $d_{2}$ is of particular theoretical and

Published by the American Physical Society under the terms of the Creative Commons Attribution 4.0 International license. Further distribution of this work must maintain attribution to the author(s) and the published article's title, journal citation, and DOI. Funded by SCOAP . phenomenological interest. For instance, the same correlations of quarks and gluons constitute the leading contribution to the Qiu-Sterman distributions [3], which play a central role in the collinear factorization of single spin asymmetries. These distributions represent the limit of vanishing impact parameter $\vec{b}_{\perp}=\overrightarrow{0}$ of the Sivers functions [4], i.e., of the transverse-momentum dependent parton distribution functions $f_{1 T}^{\perp}\left(x, \vec{b}_{\perp}, Q^{2}\right)$ that describe the distribution of an unpolarized quark inside a transversely polarized nucleon. The measurement of the Sivers distributions in polarized semi-inclusive deep-inelastic scattering and in Drell-Yan experiments is one of the main goals of the experimental programs at JLAB and the EIC. For more details, see, e.g., Refs. [5,6].

Neglecting the twist-3 contributions, $g_{2}$ can be obtained from the helicity structure function $g_{1}\left(x, Q^{2}\right)$. This involves invoking the well-known Wandzura-Wilczek relation $[7,8]$. However, a remarkable property of $g_{2}$ is that the twist-3 contribution is not power suppressed in $1 / Q$, relative to its twist-2 part. Nevertheless, its determination from longitudinally polarized deep-inelastic scattering experiments alone still represents a serious challenge and new high precision measurements are planned at JLAB and the EIC. In order to match the expected statistical precision of the planned experiments, a much improved theoretical 
understanding of higher-twist contributions is needed and $d_{2}$ is the ideal starting point.

The matrix element $d_{2}$ has another very interesting phenomenological interpretation: As was argued in Refs. $[9,10]$ it is related to the average transverse color Lorentz force acting on a quark $q$ in a nucleon which moves in the $z$ direction and is transversely polarized. More explicitly, the $y$-component of the color Lorentz force is given by

$$
\begin{aligned}
F^{q, y}(0) & =-\frac{1}{\sqrt{2} p^{+}}\left\langle p, s\left|\bar{\psi}_{q}(0) \gamma^{+} g G^{+y}(0) \psi_{q}(0)\right| p, s\right\rangle \\
& =\sqrt{2} p^{+} s^{x} d_{2}^{(q)} .
\end{aligned}
$$

Here the four-momentum $p$ of the nucleon state $|p, s\rangle$ has been chosen as $\left(p^{\mu}\right)=p^{+}(1,0,0,1) / \sqrt{2}$, the spin vector $s$ is normalized according to $s^{2}=-m_{N}^{2}, G^{\mu \nu}$ denotes the color field strength tensor and $g$ is the strong coupling constant. Using the lattice results for the proton presented in Ref. [11] (coauthored by some of us)

$$
\begin{aligned}
& d_{2}^{(u)}=0.010 \pm 0.012, \\
& d_{2}^{(d)}=-0.0056 \pm 0.0050,
\end{aligned}
$$

estimates for this force were published some time ago in Ref. [9]. It appears, however, that these estimates were affected by a misunderstanding of the respective conventions and by a sign error noted later in Ref. [10]. The corrected numbers differ by a factor $-\frac{1}{2}$ from those given in Ref. [9] and read

$$
\begin{aligned}
& F^{u, y}(0)=50 \pm 60 \mathrm{MeV} / \mathrm{fm}, \\
& F^{d, y}(0)=-28 \pm 26 \mathrm{MeV} / \mathrm{fm} .
\end{aligned}
$$

Unfortunately, the errors, which are purely statistical, are very large. Systematic uncertainties were not estimated, in particular, those arising from finite lattice spacing. Meanwhile several experiments have extracted estimates of $d_{2}^{(p)}$ and $d_{2}^{(n)}$ [12-19], where the superscript indicates proton or neutron, respectively. These estimates are found to be quite small compared to various model predictions but compatible with the old lattice results (considering the large error bars), see, e.g., Fig. 2 of Ref. [19]. (Actually, the lattice results in this figure should also have been divided by 2.) We remark that with the natural energy scale for the force $F$ being $\Lambda_{\mathrm{QCD}}^{2}$ one would not expect $d_{2}$ to be much smaller than the central values of this early lattice calculation given in Eq. (2). So there is hope that with a moderate reduction of the lattice uncertainties, this time also including systematics, one may be able to demonstrate that $d_{2}$ and thus the average color force $F$ is different from zero.

Let us stress that the experimental and lattice investigations of $d_{2}^{(p)}$ and $d_{2}^{(n)}$ are only meant to be the starting point of much broader investigations. For example, it was also argued in Ref. [10] that there exists an analogous relationship between generalized parton distributions and force distributions $\mathcal{F}_{\lambda^{\prime} \lambda}^{i}\left(\vec{b}_{\perp}\right)$ in the transverse plane,

$$
\mathcal{F}_{\lambda^{\prime} \lambda}^{i}\left(\vec{b}_{\perp}\right)=\int \frac{d^{2} \vec{\Delta}_{\perp}}{(2 \pi)^{2}} e^{-i \vec{b}_{\perp} \cdot \vec{\Delta}_{\perp}} F_{\lambda^{\prime} \lambda}^{i}\left(\vec{\Delta}_{\perp}\right)
$$

with

$$
\begin{aligned}
& F_{\lambda^{\prime} \lambda}^{i}\left(\vec{\Delta}_{\perp}\right)=-\frac{1}{\sqrt{2} p^{+}} \\
& \quad \times\left\langle p^{+}, \frac{\vec{\Delta}_{\perp}}{2}, \lambda^{\prime}\left|\bar{\psi}_{q}(0) \gamma^{+} g G^{+i}(0) \psi_{q}(0)\right| p^{+},-\frac{\vec{\Delta}_{\perp}}{2}, \lambda\right\rangle .
\end{aligned}
$$

Here $\lambda$ and $\lambda^{\prime}$ denote the nucleon polarization and $\vec{\Delta}_{\perp}$ is the transverse momentum conjugate to the impact parameter $\vec{b}_{\perp}$.

Another interesting result was derived in the very recent paper [20], where QCD factorization for quasidistributions was analyzed up to twist-3. Approaches based on so-called quasi- and pseudodistribution functions have gained prominence in lattice QCD calculations of hadron structure observables, due to their prospect of providing information that goes beyond the computation of Mellin moments of (generalized) parton distribution functions, distribution amplitudes, etc., see Refs. [21-27] and references therein.

In Ref. [20] it was shown that for quasidistributions the Wandzura-Wilczek relation $[7,8]$ is modified such that twist- 2 and twist- 3 contributions stay mixed, making their separate determination on the lattice far more difficult. Knowing $d_{2}$ from a direct lattice calculation would obviously help to unravel the different contributions.

\section{OPE AND RENORMALIZATION IN THE CONTINUUM}

A leading-order OPE (operator product expansion) analysis with massless quarks shows that the moments of $g_{1}$ and $g_{2}$ can be written as [28]

$$
\begin{aligned}
2 \int_{0}^{1} d x x^{n} g_{1}\left(x, Q^{2}\right)=\frac{1}{2} \sum_{q} Q_{q}^{2} E_{1, n}^{(q)}\left(\mu^{2} / Q^{2}, g(\mu)\right) a_{n}^{(q)}(\mu), \\
2 \int_{0}^{1} d x x^{n} g_{2}\left(x, Q^{2}\right) \\
=\frac{1}{2} \frac{n}{n+1} \sum_{q} Q_{q}^{2}\left[E_{2, n}^{(q)}\left(\mu^{2} / Q^{2}, g(\mu)\right) d_{n}^{(q)}(\mu)\right. \\
\left.\quad-E_{1, n}^{(q)}\left(\mu^{2} / Q^{2}, g(\mu)\right) a_{n}^{(q)}(\mu)\right]
\end{aligned}
$$

where $q$ runs over the light quark flavors with charges $Q_{q}$ and $\mu$ denotes the renormalization scale. Equations (6) and (7) 
hold for even $n$, with $n \geq 0$ for the former and $n \geq 2$ for the latter. The Wilson coefficients $E_{1, n}^{(q)}$ and $E_{2, n}^{(q)}$ depend on the ratio of scales $\mu^{2} / Q^{2}$ and the running coupling constant $g(\mu)$,

$$
E_{i, n}\left(\mu^{2} / Q^{2}, g(\mu)\right)=1+\mathcal{O}\left(g(\mu)^{2}\right) .
$$

To the best of our knowledge, the loop corrections for $E_{2, n}$ have not yet been calculated, while they are known up to twoloop order for $E_{1, n}$ [29]. The first-order corrections are flavor independent,

$$
E_{1, n}(1, g(\mu))=1+\frac{5}{3} \frac{g(\mu)^{2}}{16 \pi^{2}}+\mathcal{O}\left(g(\mu)^{4}\right)
$$

The reduced matrix elements $a_{n}^{(q)}(\mu)$ and $d_{n}^{(q)}(\mu)$ are defined as [28]

$$
\begin{gathered}
\left\langle p, s\left|\mathcal{O}_{\left\{\sigma \mu_{1} \cdots \mu_{n}\right\}}^{5(q)}\right| p, s\right\rangle \\
=\frac{1}{n+1} a_{n}^{(q)}\left[s_{\sigma} p_{\mu_{1}} \cdots p_{\mu_{n}}+\cdots-\text { traces }\right], \quad(10) \\
\left\langle p, s\left|\mathcal{O}_{\left[\sigma\left\{\mu_{1}\right] \mu_{2} \cdots \mu_{n}\right\}}^{5(q)}\right| p, s\right\rangle \\
=\frac{1}{n+1} d_{n}^{(q)}\left[\left(s_{\sigma} p_{\mu_{1}}-s_{\mu_{1}} p_{\sigma}\right) p_{\mu_{2}} \cdots p_{\mu_{n}}+\cdots-\text { traces }\right]
\end{gathered}
$$

in terms of matrix elements of the local operators

$$
\mathcal{O}_{\sigma \mu_{1} \cdots \mu_{n}}^{5(q)}=\left(\frac{i}{2}\right)^{n} \bar{\psi}_{q} \gamma_{\sigma} \gamma_{5} \stackrel{\leftrightarrow}{D}_{\mu_{1}} \cdots \stackrel{\leftrightarrow}{D}_{\mu_{n}} \psi_{q}-\text { traces }
$$

in the nucleon state $|p, s\rangle$. Here $\stackrel{\leftrightarrow}{D}=\vec{D}-\overleftarrow{D}$ and the symbol $\{\cdots\}([\cdots])$ indicates symmetrization (antisymmetrization) of the enclosed indices. The operator in Eq. (10) has twist two, whereas the operator in Eq. (11) has twist three. As far as the Wilson coefficients may be considered as flavor independent, we can define $a_{n}$ and $d_{n}$ for the nucleon as

$$
\begin{aligned}
& a_{n}(\mu)=\sum_{q} Q_{q}^{2} a_{n}^{(q)}(\mu), \\
& d_{n}(\mu)=\sum_{q} Q_{q}^{2} d_{n}^{(q)}(\mu) .
\end{aligned}
$$

Remarkably, in the moments (7) of $g_{2}$ the twist-3 matrix elements $d_{n}^{(q)}(\mu)$ are not suppressed relative to the twist-2 matrix elements $a_{n}^{(q)}(\mu)$.

Note that our definitions of $a_{2}$ and $d_{2}$ have been taken from Ref. [28]. In many publications alternative definitions are employed, where $a_{2}^{\text {alt }}=a_{2} / 2$ and $d_{2}^{\text {alt }}=d_{2} / 2$.

Utilizing the equations of motion of massless QCD and the relation $\left[D_{\mu}, D_{\nu}\right]=-i g G_{\mu \nu}$, the twist-3 operators $\mathcal{O}_{\left[\sigma\left\{\mu_{1}\right] \mu_{2} \cdots \mu_{n}\right\}}^{5(q)}$ can be rewritten in a manifestly interactiondependent form. For $n=2$ one finds

$\mathcal{O}_{\left[\sigma\left\{\mu_{1}\right] \mu_{2}\right\}}^{5(q)}=-\frac{g}{6} \bar{\psi}_{q}\left(\tilde{G}_{\sigma \mu_{1}} \gamma_{\mu_{2}}+\tilde{G}_{\sigma \mu_{2}} \gamma_{\mu_{1}}\right) \psi_{q}-$ traces

where $\tilde{G}^{\mu \nu}=\frac{1}{2} \epsilon^{\mu \nu \rho \sigma} G_{\rho \sigma}$ is the dual gluon field strength tensor and the totally antisymmetric $\epsilon$ tensor is such that $\epsilon^{0123}=1$. Therefore we can define the reduced matrix element $d_{2}$ in the chiral limit also by (see, e.g., Ref. [30])

$$
\begin{aligned}
-\frac{g}{6}\left\langle p, s\left|\bar{\psi}_{q}\left(\tilde{G}_{\sigma \mu_{1}} \gamma_{\mu_{2}}+\tilde{G}_{\sigma \mu_{2}} \gamma_{\mu_{1}}\right) \psi_{q}-\operatorname{traces}\right| p, s\right\rangle \\
=\frac{1}{3} d_{2}^{(q)}\left[\left(s_{\sigma} p_{\mu_{1}}-s_{\mu_{1}} p_{\sigma}\right) p_{\mu_{2}}+\cdots-\text { traces }\right] .
\end{aligned}
$$

The Wilson coefficients (8) can be computed in perturbation theory, while the nucleon matrix elements $a_{n}^{(q)}$ and $d_{n}^{(q)}$ are nonperturbative quantities. For simplicity, in the following we omit the flavor indices, in most cases.

The renormalization of the operators which contribute to the moments of $g_{2}$ has been studied by several authors in continuum perturbation theory [31-38]. For example, in Refs. [37,38] the following operators are considered for $n=2$ in the flavor-nonsinglet sector:

$$
\begin{gathered}
R_{F}^{\sigma \mu \nu}=-\frac{i^{2}}{3}\left[2 \bar{\psi} \gamma^{\sigma} \gamma_{5} D^{\{\mu} D^{\nu\}} \psi-\bar{\psi} \gamma^{\mu} \gamma_{5} D^{\{\sigma} D^{\nu\}} \psi-\bar{\psi} \gamma^{\nu} \gamma_{5} D^{\{\mu} D^{\sigma\}} \psi\right]-\text { traces, } \\
R_{1}^{\sigma \mu \nu}=\frac{1}{12} g\left[\epsilon^{\sigma \mu \alpha \beta} \bar{\psi} G_{\alpha \beta} \gamma^{\nu} \psi+\epsilon^{\sigma \nu \alpha \beta} \bar{\psi} G_{\alpha \beta} \gamma^{\mu} \psi\right]-\text { traces, } \\
R_{m}^{\sigma \mu \nu}=-i m \bar{\psi} \gamma^{\sigma} \gamma_{5} D^{\{\mu} \gamma^{\nu\}} \psi-\text { traces, } \\
R_{\mathrm{eq}}^{\sigma \mu \nu}=-\frac{i}{3}\left[\bar{\psi} \gamma^{\sigma} \gamma_{5} D^{\{\mu} \gamma^{\nu\}}(i \not D-m) \psi+\bar{\psi}(i \not D-m) \gamma^{\sigma} \gamma_{5} D^{\{\mu} \gamma^{\nu\}} \psi\right]-\text { traces. }
\end{gathered}
$$

The gluon field strength tensor $G_{\alpha \beta}$ could alternatively be expressed in terms of a commutator of two covariant derivatives. As Eqs. (11) and (12) show, the matrix element $d_{2}$ corresponds to the nucleon matrix elements of the renormalized operators (17). The operators (17)-(20) are linearly dependent, 


$$
R_{F}^{\sigma \mu \nu}=\frac{2}{3} R_{m}^{\sigma \mu \nu}+R_{1}^{\sigma \mu \nu}+R_{\mathrm{eq}}^{\sigma \mu \nu} .
$$

In the massless case, this relation leads to Eq. (15) upon application of the equations of motion.

Calculating the quark-quark-gluon three-point functions with a single insertion of each of these operators in one-loop perturbation theory, one sees that also a gauge-variant operator has to be taken into account in the process of renormalization,

$$
R_{\text {eq1 }}^{\sigma \mu \nu}=-\frac{i}{3}\left[\bar{\psi} \gamma^{\sigma} \gamma_{5} \partial^{\{\mu} \gamma^{\nu\}}(i \not D-m) \psi+\bar{\psi}(i \not D-m) \gamma^{\sigma} \gamma_{5} \partial^{\{\mu} \gamma^{\nu\}} \psi\right]-\text { traces. }
$$

Of course, in physical matrix elements neither $R_{\text {eq }}$ nor $R_{\text {eq1 }}$ will contribute. They show up, however, in off-shell vertex functions and influence the renormalization factors.

\section{LATTICE OPERATORS AND RENORMALIZATION}

In the following, we use Euclidean notation. For our lattice evaluation of the reduced matrix elements $a_{2}$ and $d_{2}$ we construct discretized versions of the relevant operators. In the process of the renormalization of these operators, operator mixing requires particular attention because the discrete symmetry group $H(4)$ of a hypercubic lattice is less restrictive than the continuous symmetry group $O(4)$ of Euclidean spacetime.

In the case of the twist- 2 matrix element $a_{2}$ we use the four-dimensional multiplet of operators spanned by

$$
\mathcal{O}_{\{234\}}^{5}, \mathcal{O}_{\{134\}}^{5}, \mathcal{O}_{\{124\}}^{5}, \mathcal{O}_{\{123\}}^{5},
$$

where

$$
\mathcal{O}_{\sigma \mu \nu}^{5}=\bar{\psi} \gamma_{\sigma} \gamma_{5} \stackrel{\leftrightarrow}{D}_{\mu} \stackrel{\leftrightarrow}{D}_{\nu} \psi
$$

The operators (23) transform according to the representation $\tau_{3}^{(4)}$ of the hypercubic group H(4) [39-41]. They have mass dimension five and charge conjugation parity +1 . These properties ensure that they do not mix with any other gauge-invariant operators of the same or lower dimension. Therefore, they are multiplicatively renormalizable. We take the corresponding renormalization factor from Table XI of Ref. [42].

For the evaluation of the twist- 3 matrix element $d_{2}$ we use multiplets of operators with charge conjugation parity +1 which transform under the hypercubic group according to the representation $\tau_{1}^{(8)}$. Among the gauge-invariant operators of dimension $\leq 5$ there are three multiplets that have these symmetry properties and can therefore mix with each other under renormalization. Suitable bases transforming according to the same (not just equivalent) unitary representation of $H(4)$ are

$$
\begin{aligned}
& \mathcal{O}_{1}^{(1)}=\frac{1}{4 \sqrt{3}}\left(2 \mathcal{O}_{2\{14\}}^{5}-\mathcal{O}_{1\{24\}}^{5}-\mathcal{O}_{4\{12\}}^{5}\right), \\
& \mathcal{O}_{1}^{(2)}=\frac{1}{4 \sqrt{3}}\left(2 \mathcal{O}_{2\{13\}}^{5}-\mathcal{O}_{1\{23\}}^{5}-\mathcal{O}_{3\{12\}}^{5}\right), \\
& \mathcal{O}_{1}^{(3)}=\frac{1}{4 \sqrt{3}}\left(2 \mathcal{O}_{3\{14\}}^{5}-\mathcal{O}_{1\{34\}}^{5}-\mathcal{O}_{4\{13\}}^{5}\right), \\
& \mathcal{O}_{1}^{(4)}=\frac{1}{4 \sqrt{3}}\left(2 \mathcal{O}_{3\{24\}}^{5}-\mathcal{O}_{2\{34\}}^{5}-\mathcal{O}_{4\{23\}}^{5}\right), \\
& \mathcal{O}_{1}^{(5)}=\frac{1}{4}\left(\mathcal{O}_{4\{23\}}^{5}-\mathcal{O}_{2\{34\}}^{5}\right), \\
& \mathcal{O}_{1}^{(6)}=\frac{1}{4}\left(\mathcal{O}_{4\{13\}}^{5}-\mathcal{O}_{1\{34\}}^{5}\right), \\
& \mathcal{O}_{1}^{(7)}=\frac{1}{4}\left(\mathcal{O}_{4\{12\}}^{5}-\mathcal{O}_{1\{24\}}^{5}\right), \\
& \mathcal{O}_{1}^{(8)}=\frac{1}{4}\left(\mathcal{O}_{3\{12\}}^{5}-\mathcal{O}_{1\{23\}}^{5}\right),
\end{aligned}
$$

$$
\begin{aligned}
& \mathcal{O}_{2}^{(1)}=\frac{1}{2 \sqrt{2}} \bar{\psi}\left(\gamma_{3} \gamma_{1} \stackrel{\leftrightarrow}{D}_{1}-\gamma_{3} \gamma_{4} \stackrel{\leftrightarrow}{D}_{4}\right), \\
& \mathcal{O}_{2}^{(2)}=\frac{1}{2 \sqrt{2}} \bar{\psi}\left(\gamma_{4} \gamma_{3} \stackrel{\leftrightarrow}{D}_{3}-\gamma_{4} \gamma_{1} \stackrel{\leftrightarrow}{D}_{1}\right), \\
& \mathcal{O}_{2}^{(3)}=\frac{1}{2 \sqrt{2}} \bar{\psi}\left(\gamma_{2} \gamma_{4} \stackrel{\leftrightarrow}{D}_{4}-\gamma_{2} \gamma_{1} \stackrel{\leftrightarrow}{D}_{1}\right), \\
& \mathcal{O}_{2}^{(4)}=\frac{1}{2 \sqrt{2}} \bar{\psi}\left(\gamma_{1} \gamma_{2} \stackrel{\leftrightarrow}{D}_{2}-\gamma_{1} \gamma_{4} \stackrel{\leftrightarrow}{D}_{4}\right), \\
& \mathcal{O}_{2}^{(5)}=\frac{1}{2 \sqrt{6}} \bar{\psi}\left(2 \gamma_{1} \gamma_{3} \stackrel{\leftrightarrow}{D}_{3}-\gamma_{1} \gamma_{2} \stackrel{\leftrightarrow}{D}_{2}-\gamma_{1} \gamma_{4} \stackrel{\leftrightarrow}{D}_{4}\right) \psi \\
& \mathcal{O}_{2}^{(6)}=\frac{1}{2 \sqrt{6}} \bar{\psi}\left(-2 \gamma_{2} \gamma_{3} \stackrel{\leftrightarrow}{D}_{3}+\gamma_{2} \gamma_{1} \stackrel{\leftrightarrow}{D}_{1}+\gamma_{2} \gamma_{4} \stackrel{\leftrightarrow}{D}_{4}\right) \psi \\
& \mathcal{O}_{2}^{(7)}=\frac{1}{2 \sqrt{6}} \bar{\psi}\left(2 \gamma_{3} \gamma_{2} \stackrel{\leftrightarrow}{D}_{2}-\gamma_{3} \gamma_{1} \stackrel{\leftrightarrow}{D}_{1}-\gamma_{3} \gamma_{4} \stackrel{\leftrightarrow}{D}_{4}\right) \psi, \\
& \mathcal{O}_{2}^{(8)}=\frac{1}{2 \sqrt{6}} \bar{\psi}\left(-2 \gamma_{4} \gamma_{2} \stackrel{\leftrightarrow}{D}_{2}+\gamma_{4} \gamma_{1} \stackrel{\leftrightarrow}{D}_{1}+\gamma_{4} \gamma_{3} \stackrel{\leftrightarrow}{D}_{3}\right) \psi,
\end{aligned}
$$




$$
\begin{aligned}
& \mathcal{O}_{3}^{(1)}=\bar{\psi}\left(\gamma_{1} \stackrel{\leftrightarrow}{D}_{[3} \stackrel{\leftrightarrow}{D}_{1]}-\gamma_{4} \stackrel{\leftrightarrow}{D}_{[3} \stackrel{\leftrightarrow}{D}_{4]}\right), \\
& \mathcal{O}_{3}^{(2)}=\bar{\psi}\left(\gamma_{3} \stackrel{\leftrightarrow}{D}_{[4} \stackrel{\leftrightarrow}{D}_{3]}-\gamma_{1} \stackrel{\leftrightarrow}{D}_{[4} \stackrel{\leftrightarrow}{D}_{1]}\right), \\
& \mathcal{O}_{3}^{(3)}=\bar{\psi}\left(\gamma_{4} \stackrel{\leftrightarrow}{D}_{[2} \stackrel{\leftrightarrow}{D}_{4]}-\gamma_{1} \stackrel{\leftrightarrow}{D}_{[2} \stackrel{\leftrightarrow}{D}_{1]}\right), \\
& \mathcal{O}_{3}^{(4)}=\bar{\psi}\left(\gamma_{2} \stackrel{\leftrightarrow}{D}_{[1} \stackrel{\leftrightarrow}{D}_{2]}-\gamma_{4} \stackrel{\leftrightarrow}{D}_{[1} \stackrel{\leftrightarrow}{D}_{4]}\right), \\
& \mathcal{O}_{3}^{(5)}=\frac{1}{\sqrt{3}} \bar{\psi}\left(2 \gamma_{3} \stackrel{\leftrightarrow}{D}_{[1} \stackrel{\leftrightarrow}{D}_{3]}-\gamma_{2} \stackrel{\leftrightarrow}{D}_{[1} \stackrel{\leftrightarrow}{D}_{2]}-\gamma_{4} \stackrel{\leftrightarrow}{D}_{[1} \stackrel{\leftrightarrow}{D}_{4]}\right) \psi, \\
& \mathcal{O}_{3}^{(6)}=\frac{1}{\sqrt{3}} \bar{\psi}\left(-2 \gamma_{3} \stackrel{\leftrightarrow}{D}_{[2} \stackrel{\leftrightarrow}{D}_{3]}+\gamma_{1} \stackrel{\leftrightarrow}{D}_{[2} \stackrel{\leftrightarrow}{D}_{1]}+\gamma_{4} \stackrel{\leftrightarrow}{D}_{[2} \stackrel{\leftrightarrow}{D}_{4]}\right) \psi, \\
& \mathcal{O}_{3}^{(7)}=\frac{1}{\sqrt{3}} \bar{\psi}\left(2 \gamma_{2} \stackrel{\leftrightarrow}{D}_{[3} \stackrel{\leftrightarrow}{D}_{2]}-\gamma_{1} \stackrel{\leftrightarrow}{D}_{[3} \stackrel{\leftrightarrow}{D}_{1]}-\gamma_{4} \stackrel{\leftrightarrow}{D}_{[3} \stackrel{\leftrightarrow}{D}_{4]}\right) \psi, \\
& \mathcal{O}_{3}^{(8)}=\frac{1}{\sqrt{3}} \bar{\psi}\left(-2 \gamma_{2} \stackrel{\leftrightarrow}{D}_{[4} \stackrel{\leftrightarrow}{D}_{2]}+\gamma_{1} \stackrel{\leftrightarrow}{D}_{[4} \stackrel{\leftrightarrow}{D}_{1]}+\gamma_{3} \stackrel{\leftrightarrow}{D}_{[4} \stackrel{\leftrightarrow}{D}_{3]}\right) \psi
\end{aligned}
$$

The lattice operators (25) are Euclidean counterparts of the Minkowski operators (17), while the operators (27) correspond to the operators (18) with the field strength expressed in terms of a commutator of two covariant derivatives. The operators (26) are analogous to (19). Under renormalization all three multiplets are expected to mix with each other. In the continuum, $R_{m}$ disappears in the chiral limit. On the lattice, the explicit breaking of chiral symmetry caused by Wilson-type fermions persists even for massless quarks. Therefore $\mathcal{O}_{2}^{(i)}$ will contribute with a coefficient $\propto a^{-1}$, where $a$ denotes the lattice spacing. The operators $\mathcal{O}_{3}^{(i)}$, on the contrary, are of the same dimension as $\mathcal{O}_{1}^{(i)}$ and mix with a coefficient of order $g^{2}$, which should be small. The same holds for lattice counterparts of $R_{\text {eq }}$ and $R_{\text {eq1 }}$. Hence, in a first approximation we take into account only the multiplets (25) and (26). Their renormalization and mixing can be treated along the lines of Ref. [42], provided one multiplies the operators (26) with $1 / a$. Both operator multiplets then have dimension five.

The renormalized operators of the multiplet (25) are now given by

$$
\mathcal{O}_{1}^{(i) \mathrm{R}}=\hat{Z}_{11}(\mu, a) \mathcal{O}_{1}^{(i)}+\frac{1}{a} \hat{Z}_{12}(\mu, a) \mathcal{O}_{2}^{(i)}
$$

Here we stick to the notation of Ref. [42], where $\hat{Z}_{m m^{\prime}}(\mu, a)$ denotes the renormalization and mixing matrix in the nonperturbative scheme used on the lattice. For this scheme we choose the RI'-MOM (regularization independent momentum subtraction) scheme, i.e., the operators are taken at vanishing momentum. In order to suppress powerlike lattice artifacts as far as possible the external quark momenta are chosen as

$$
\frac{\mu}{2}(1,1,1,1)
$$

with the renormalization scale $\mu$. Presently we cannot convert the coefficients $\hat{Z}_{m m^{\prime}}(\mu, a)$ (and hence the renormalized operators) to the $\overline{\mathrm{MS}}$ scheme, because the required perturbative calculations in the continuum are not yet available. Our procedure accounts for the mixing with lower-dimensional operators caused by the explicit breakdown of chiral symmetry in our simulations, but further mixing effects are still neglected.

In the chiral limit, the matrix element $d_{2}$ is multiplicatively renormalizable [31]. Rewriting Eq. (28) as

$$
\mathcal{O}_{1}^{(i) \mathrm{R}}=\hat{Z}_{11}(\mu, a)\left(\mathcal{O}_{1}^{(i)}+\frac{1}{a} \frac{\hat{Z}_{12}(\mu, a)}{\hat{Z}_{11}(\mu, a)} \mathcal{O}_{2}^{(i)}\right),
$$

we see that $\mathcal{O}_{1}^{(i) \mathrm{R}}$ will have a multiplicative dependence on $\mu$ if the ratio $\hat{Z}_{12}(\mu, a) / \hat{Z}_{11}(\mu, a)$ does not depend on $\mu$. It turns out that this requirement is better fulfilled when we use instead of the $2 \times 2$ matrix $\hat{Z}(\mu, a)$ the matrix $\tilde{Z}(\mu, a)$ constructed in the following way, cf. Ref. [43]. We compute $\hat{Z}(\mu, a) \hat{Z}^{-1}\left(\mu_{0}, a\right)$ for the renormalization scales $\mu$ of interest and a reference scale $\mu_{0}$ chosen as $\mu_{0}=2 \mathrm{GeV}$. Within our approximations, this matrix should have a continuum limit, which we evaluate by fitting the lattice spacing dependence with a quadratic polynomial in $a^{2}$. Denoting the result $R\left(\mu, \mu_{0}\right)$, we define

$$
\tilde{Z}(\mu, a)=R\left(\mu, \mu_{0}\right) \hat{Z}\left(\mu_{0}, a\right) .
$$

To improve on this would require the consideration of quark-quark-gluon matrix elements instead of quark-quark matrix elements.

\section{SIMULATION DETAILS}

\section{A. Lattice setup}

To compute the reduced matrix elements $a_{n}^{(f)}(\mu)$ and $d_{n}^{(f)}(\mu)$ in (10) and (11) for $n=2$ we analyze a subset of the lattice gauge ensembles generated within the Coordinated Lattice Simulations (CLS) effort [44]. The ensembles have been generated using a tree-level Symanzik improved gauge action with $N_{f}=2+1$ flavors of nonperturbatively $O(a)$ improved Wilson (clover) fermions. Near zero modes of the Wilson-Dirac operator are avoided by applying twisted-mass determinant reweighting to achieve stable Monte Carlo sampling [45]. Furthermore we improve the overlap of the interpolating currents at the source/sink time slice using Wuppertal smeared quarks [46] in the source/sink interpolators with APE smoothed spatial gauge links [47].

In most of our simulations we use open boundary conditions in time. Especially for the very fine lattices this avoids freezing of the topological charge and large autocorrelation times [45,48]. In order to suppress the distortions caused by the loss of translation invariance in time we restrict our measurements to regions with 
TABLE I. CLS gauge ensembles analyzed in this work. The ensemble identifier is given in the first column, followed by the inverse gauge coupling $\beta$ and the lattice size. The column "BC" indicates whether the boundary condition in time was open (o) or (anti)periodic (p). In the next columns we give the lattice spacing $a$, the pion mass $m_{\pi}$ and the product of the spatial lattice size $L=a N_{s}$ with the pion mass. The column " $t / a$ " contains the list of source-sink distances analyzed on this lattice. The subscript \#meas specifies how many measurements have been performed for the respective source-sink distance. In physical units these distances roughly correspond to 0.9 , 1.0 and $1.2 \mathrm{fm}$. The number of analyzed configurations is given in the column " $n_{\mathrm{cnfgs}}$," and "Trajectory" specifies the trajectory in the quark mass plane to which the ensemble belongs.

\begin{tabular}{|c|c|c|c|c|c|c|c|c|c|}
\hline Ensemble & $\beta$ & $N_{s}^{3} \times N_{t}$ & $\mathrm{BC}$ & $a(\mathrm{fm})$ & $m_{\pi}(\mathrm{MeV})$ & $L m_{\pi}$ & $t / a_{\# \text { \#meas }}$ & $n_{\text {cnfgs }}$ & Trajectory \\
\hline A654 & 3.34 & $24^{3} \times 48$ & $\mathrm{p}$ & 0.0984 & 334 & 4.0 & $9_{4}, 11_{4}, 13_{4}$ & 2534 & trm \\
\hline A653 & 3.34 & $24^{3} \times 48$ & $\mathrm{p}$ & 0.0984 & 426 & 5.1 & $94,11_{4}, 13_{4}$ & 2525 & trm, symm \\
\hline H106 & 3.4 & $32^{3} \times 96$ & o & 0.0859 & 272 & 3.8 & $10_{2}, 12_{3}, 14_{4}$ & 1544 & $\mathrm{msc}$ \\
\hline H105 & 3.4 & $32^{3} \times 96$ & o & 0.0859 & 279 & 3.9 & $10_{2}, 12_{3}, 14_{4}$ & 2065 & trm \\
\hline H102 & 3.4 & $32^{3} \times 96$ & o & 0.0859 & 352 & 4.9 & $10_{2}, 12_{3}, 14_{4}$ & 2005 & trm \\
\hline H107 & 3.4 & $32^{3} \times 96$ & o & 0.0859 & 366 & 5.1 & $10_{2}, 12_{3}, 14_{4}$ & 1561 & $\mathrm{msc}$ \\
\hline H101 & 3.4 & $32^{3} \times 96$ & o & 0.0859 & 420 & 5.9 & $10_{2}, 12_{2}, 14_{2}$ & 2016 & trm, symm \\
\hline D451 & 3.46 & $64^{3} \times 128$ & $\mathrm{p}$ & 0.0760 & 217 & 5.4 & $11_{4}, 13_{4}, 16_{4}$ & 531 & $\mathrm{msc}$ \\
\hline N450 & 3.46 & $48^{3} \times 128$ & $\mathrm{p}$ & 0.0760 & 285 & 5.3 & $11_{4}, 13_{4}, 16_{4}$ & 1129 & $\mathrm{msc}$ \\
\hline B452 & 3.46 & $32^{3} \times 64$ & $\mathrm{p}$ & 0.0760 & 350 & 4.3 & $11_{4}, 13_{4}, 16_{4}$ & 1941 & $\mathrm{msc}$ \\
\hline S400 & 3.46 & $32^{3} \times 128$ & o & 0.0760 & 352 & 4.3 & $11_{4}, 13_{4}, 16_{4}$ & 2872 & trm \\
\hline B450 & 3.46 & $32^{3} \times 64$ & $\mathrm{p}$ & 0.0760 & 418 & 5.2 & $11_{4}, 13_{4}, 16_{4}$ & 1612 & trm, symm \\
\hline N201 & 3.55 & $48^{3} \times 128$ & o & 0.0643 & 285 & 4.5 & $14_{2}, 16_{3}, 19_{4}$ & 1520 & $\mathrm{msc}$ \\
\hline N203 & 3.55 & $48^{3} \times 128$ & o & 0.0643 & 345 & 5.4 & $14_{2}, 16_{3}, 19_{4}$ & 1543 & trm \\
\hline N204 & 3.55 & $48^{3} \times 128$ & o & 0.0643 & 351 & 5.5 & $14_{2}, 16_{3}, 19_{4}$ & 1500 & $\mathrm{msc}$ \\
\hline N202 & 3.55 & $48^{3} \times 128$ & o & 0.0643 & 411 & 6.4 & $14_{2}, 16_{2}, 19_{4}$ & 899 & trm, symm \\
\hline J304 & 3.7 & $64^{3} \times 192$ & o & 0.0497 & 260 & 4.2 & $17_{3}, 21_{3}, 24_{4}$ & 1630 & $\mathrm{msc}$ \\
\hline N302 & 3.7 & $48^{3} \times 128$ & o & 0.0497 & 346 & 4.2 & $17_{2}, 21_{3}, 24_{4}$ & 2201 & trm \\
\hline N304 & 3.7 & $48^{3} \times 128$ & o & 0.0497 & 351 & 4.3 & $17_{2}, 21_{3}, 24_{4}$ & 1652 & $\mathrm{msc}$ \\
\hline N300 & 3.7 & $48^{3} \times 128$ & o & 0.0497 & 422 & 5.1 & $17_{2}, 21_{2}, 24_{4}$ & 500 & trm, symm \\
\hline J501 & 3.85 & $64^{3} \times 192$ & o & 0.0391 & 333 & 4.2 & $22_{2}, 27_{3}, 32_{4}$ & 750 & trm \\
\hline
\end{tabular}

sufficiently large distances from the temporal boundaries, see, e.g., Refs. [42,49]. Only a few of the coarser lattices have been simulated using (anti)periodic boundary conditions. An overview of the gauge ensembles used in this work is given in Table I. They have been generated along three different trajectories in the quark mass plane, which are indicated in the last column of the table. Along the trajectory labeled by "trm," the trace of the quark mass matrix is held constant, approximately equal to its physical value [44]. Along the trajectory labeled by "msc," the renormalized strange quark mass is set to its physical value [50], and the symmetric line with equal masses of the light quarks and the strange quark is labeled by "symm." A general explanation of this strategy can be found in [50]. In summary we use six different lattice spacings ranging from about 0.039 up to $0.098 \mathrm{fm}$ and $m_{\pi}$ goes down from $\sim 420$ to $\sim 220 \mathrm{MeV}$. With linear spatial lattice extents $L m_{\pi}=a N_{s} m_{\pi}$ between 3.8 and 6.4, finite volume effects are expected to be moderate. Removing the leading $O(a)$ discretization effects in the relevant matrix elements requires Symanzik improvement of the corresponding operators. This has not been implemented in our study.
The extraction of the reduced matrix elements $a_{n}^{(f)}(\mu)$ and $d_{n}^{(f)}(\mu)$ relies on the computation of ratios between three- and two-point functions. The evaluation of the two-point functions requires only the inversion of the lattice Dirac operator by means of common numerical solvers. In particular, we use a modified version of the Wuppertal adaptive algebraic multigrid code DD- $\alpha$ AMG $[51,52]$ on the Xeon Phi architecture [53-56] and the IDFLS solver [57,58] on $x 86-64$. The three-point functions are computed with the help of the sequential source method [59], extensively applying the socalled coherent sink method used by the LHPC Collaboration in Ref. [60]. All the computations are performed using the CHROMA software package [61] and additional libraries implemented by our group.

\section{B. Correlation functions}

In Sec. II we used the OPE to relate the moments of the structure functions $g_{i}\left(x, Q^{2}\right)$ to the reduced matrix elements $a_{n}^{(f)}(\mu)$ and $d_{n}^{(f)}(\mu)$ and specified their definitions in Eqs. (10) and (11). The corresponding matrix elements are extracted on the lattice from two- and three-point functions of the form 


$$
\begin{aligned}
C_{2 \mathrm{pt}}^{\vec{p}}(t)= & P_{+}^{\alpha \beta} C_{2 \mathrm{pt}, \beta \alpha}^{\vec{p}}(t) \\
= & a^{3} \sum_{\vec{x}} e^{-i \vec{p} \cdot \vec{x}} P_{+}^{\alpha \beta}\left\langle\mathcal{N}^{\beta}(\vec{x}, t) \overline{\mathcal{N}}^{\alpha}(\overrightarrow{0}, 0)\right\rangle, \\
C_{3 \mathrm{pt}, \Gamma}^{\vec{p}, \vec{p}^{\prime}, \mathcal{O}}(t, \tau)= & \Gamma^{\alpha \beta} C_{3 \mathrm{pt}, \vec{p}^{\prime}, \mathcal{O} \alpha}(t, \tau) \\
= & a^{6} \sum_{\vec{x}, \vec{y}} e^{-i \vec{p}^{\prime} \cdot \vec{x}+i\left(\vec{p}^{\prime}-\vec{p}\right) \cdot \vec{y}} \\
& \quad \times \Gamma^{\alpha \beta}\left\langle\mathcal{N}^{\beta}(\vec{x}, t) \mathcal{O}(\vec{y}, \tau) \overline{\mathcal{N}}^{\alpha}(\overrightarrow{0}, 0)\right\rangle .
\end{aligned}
$$

The initial (final) momentum is denoted by $\vec{p}\left(\vec{p}^{\prime}\right)$. The quantities of interest in this work allow us to restrict the kinematics to the forward limit, thus we use $\vec{p}=\vec{p}^{\prime}$ from now on. The nucleon is created by the interpolating current $\overline{\mathcal{N}}$ at the source time slice $t_{\text {src }}=0$ and annihilated at the sink time slice $t$. In the case of the three-point correlation function an additional local current $\mathcal{O}$ is inserted at the time slice $\tau$ with $t>\tau>0$. The nucleon interpolating current is defined by

$$
\mathcal{N}^{\alpha}(\vec{x}, t)=\left(u(\vec{x}, t)^{T} C \gamma_{5} d(\vec{x}, t)\right) u^{\alpha}(\vec{x}, t),
$$

where $C$ is the charge conjugation matrix and the quark fields are smeared separately in all spatial directions using the techniques mentioned in Sec. IV A. Furthermore, we define the positive parity projector $P_{+}=\left(1+\gamma_{4}\right) / 2$, and $\Gamma=P_{+}(-i) \gamma_{j} \gamma_{5}$ corresponds to the difference between the two spin projections with respect to the direction $j=1$, 2, 3.

When evaluating the three-point functions we consider quark-line connected diagrams only. Calculating the quark-line disconnected diagrams is computationally very expensive, but probably only of secondary importance for the physical quantities such as the color Lorentz force on a quark in a nucleon. However, we should keep in mind that, strictly speaking, only flavor-nonsinglet quantities like $d_{2}^{(u)}-d_{2}^{(d)}$ are free of quark-line disconnected contributions.

The correlation functions are related to matrix elements by inserting complete sets of energy eigenstates. In the limit of large Euclidean times $t, \tau$ and $t-\tau$ excited states are exponentially suppressed and the correlation functions can be approximated by the ground-state contribution,

$$
\begin{aligned}
C_{2 \mathrm{pt}}^{\vec{p}}(t) \approx \sum_{\sigma} & P_{+}^{\alpha \beta}\left\langle 0\left|\mathcal{N}^{\beta}\right| N_{\sigma}^{\vec{p}}\right\rangle\left\langle N_{\sigma}^{\vec{p}}\left|\overline{\mathcal{N}}^{\alpha}\right| 0\right\rangle \frac{e^{-E_{\vec{p}} t}}{2 E_{\vec{p}}}, \\
C_{3 \mathrm{pt}, \Gamma}^{\vec{p}, \mathcal{O}}(t, \tau) \approx & \sum_{\sigma, \sigma^{\prime}} \Gamma^{\alpha \beta}\left\langle 0\left|\mathcal{N}^{\beta}\right| N_{\sigma^{\prime}}^{\vec{p}}\right\rangle \\
& \times\left\langle N_{\sigma^{\prime}}^{\vec{p}}|\mathcal{O}| N_{\sigma}^{\vec{p}}\right\rangle\left\langle N_{\sigma}^{\vec{p}}\left|\overline{\mathcal{N}}^{\alpha}\right| 0\right\rangle \frac{e^{-E_{\vec{p}} t}}{4 E_{\vec{p}}^{2}},
\end{aligned}
$$

where $\left|N_{\sigma}^{\vec{p}}\right\rangle$ denotes a nucleon state with spin projection $\sigma$ and momentum $\vec{p}$. The overlap matrix elements can be written as

$$
P_{+}^{\alpha \beta}\left\langle 0\left|\mathcal{N}^{\beta}(\overrightarrow{0}, 0)\right| N_{\sigma}^{\vec{p}}\right\rangle=P_{+}^{\alpha \beta} \sqrt{Z_{\vec{p}}} u_{\sigma}^{\vec{p}, \beta}
$$

in terms of the momentum and smearing-dependent overlap factors $Z_{\vec{p}}$ and the nucleon spinor $u_{\sigma}^{\vec{p}, \beta}$. Similarly, the matrix elements of $\mathcal{O}$ can be expressed in the form

$$
\left\langle N_{\sigma^{\prime}}^{\vec{p}}|\mathcal{O}| N_{\sigma}^{\vec{p}}\right\rangle=\bar{u}_{\sigma^{\prime}}^{\vec{p}} J[\mathcal{O}] u_{\sigma}^{\vec{p}}
$$

Using the spinor identity $\sum_{\sigma} u_{\sigma}^{\vec{p}} \overrightarrow{u_{\sigma}^{p}}=E_{\vec{p}} \gamma_{4}-$ $i \vec{p} \cdot \vec{\gamma}+m_{N}$, we rewrite (35) and (36) as

$$
\begin{gathered}
C_{2 \mathrm{pt}}^{\vec{p}}(t)=Z_{\vec{p}} \frac{E_{\vec{p}}+m_{N}}{E_{\vec{p}}} e^{-E_{\vec{p}} t}+\cdots, \\
C_{3 \mathrm{p}, \Gamma}^{\vec{p}, \mathcal{O}}(t, \tau)=Z_{\vec{p}} e^{-E_{\vec{p}} t} B_{\Gamma, \mathcal{O}}^{\vec{p}}+\cdots,
\end{gathered}
$$

where

$$
\begin{aligned}
B_{\Gamma, \mathcal{O}}^{\vec{p}}= & \frac{1}{4 E_{\vec{p}}^{2}} \operatorname{Tr}\left\{\Gamma\left(E_{\vec{p}} \gamma_{4}-i \vec{p} \cdot \vec{\gamma}+m_{N}\right) J[\mathcal{O}]\right. \\
& \left.\times\left(E_{\vec{p}} \gamma_{4}-i \vec{p} \cdot \vec{\gamma}+m_{N}\right)\right\} .
\end{aligned}
$$

The relations between the ground-state matrix elements $\left\langle N_{\sigma^{\prime}}^{\vec{p}}|\mathcal{O}| N_{\sigma}^{\vec{p}}\right\rangle$ and the reduced matrix elements are given in Sec. II. However, in addition to the ground-state contributions we have to take into account possible excited states in Eqs. (39) and (40). An analysis of the first excited-state contribution is given in the next subsection.

\section{Excited-state contributions}

In the two- and three-point functions (39) and (40), respectively, the signal-to-noise ratio decreases exponentially with the source-sink separation in time. However, for small time distances between the operators we still find significant excited-state contributions. We take these contributions into account by including excited-state terms in the spectral decomposition of the correlation functions. Our ansatz reads

$$
\begin{aligned}
C_{2 \mathrm{pt}}^{\vec{p}}(t) \approx & Z_{\vec{p}} \frac{E_{\vec{p}}+m_{N}}{E_{\vec{p}}} e^{-E_{\vec{p}} t}\left(1+A e^{-\Delta E_{\vec{p}} t}\right), \\
C_{3 \mathrm{pt}, \Gamma}^{\vec{p}, \mathcal{O}}(t, \tau) \approx & Z_{\vec{p}} e^{-E_{\vec{p}} t} B_{\Gamma, \mathcal{O}}^{\vec{p}}\left(1+B_{10} e^{-\Delta E_{\vec{p}}(t-\tau)}\right. \\
& \left.+B_{01} e^{-\Delta E_{\vec{p}} \tau}+B_{11} e^{-\Delta E_{\vec{p}} t}\right) .
\end{aligned}
$$

Here $\Delta E_{\vec{p}}$ denotes the energy difference between the first excited state and the ground state, which is taken to be the 
same in both correlators. The amplitudes of the excitedstate contributions in the two- and three-point functions are denoted by $A$ and $B$, respectively. All amplitudes depend on the smearing and on the momentum of the interpolating currents at the source and the sink, while $B_{10}, B_{01}$ and $B_{11}$ also depend on the inserted current $\mathcal{O}$ and the spin matrix $\Gamma$. Since we only consider the forward limit, we may set $B_{10}=B_{01}$.

\section{Ratios}

Instead of performing a simultaneous fit to the two- and three-point functions, we consider the two-point functions along with ratios of three-point functions divided by twopoint functions,

$$
R_{\mathcal{O}, \Gamma}^{\vec{p}}=\frac{C_{3 \mathrm{pt}, \Gamma}^{\vec{p}, \mathcal{O}}(t, \tau)}{C_{2 \mathrm{pt}}^{\vec{p}}(t)} \stackrel{\overbrace \gg \tau \gg 0}{\approx} \frac{E_{\vec{p}}}{E_{\vec{p}}+m_{N}} B_{\Gamma, \mathcal{O}}^{\vec{p}} .
$$

In such a ratio the leading-order time dependence and the overlap factors are eliminated and the ground-state contribution corresponds directly to the matrix element we are interested in. Taking into account excited-state contributions according to Eqs. (42) and (43), we would arrive at the fit ansatz

$$
\begin{aligned}
R_{\mathcal{O}, \Gamma}^{\vec{p}} \approx & \frac{E_{\vec{p}}}{E_{\vec{p}}+m_{N}} B_{\Gamma, \mathcal{O}}^{\vec{p}} \\
& \times \frac{1+B_{10} e^{-\Delta E_{\vec{p}}(t-\tau)}+B_{01} e^{-\Delta E_{\vec{p}} \tau}+B_{11} e^{-\Delta E_{\vec{p}} t}}{1+A e^{-\Delta E_{\vec{p}} t}} .
\end{aligned}
$$

We assume that the ground-state energies are well described by the continuum dispersion relation

$$
E_{\vec{p}}=\sqrt{\vec{p}^{2}+m_{N}^{2}}
$$

in our fitting analysis. This need not be the case for $\Delta E_{\vec{p}}$ since in general multihadron states may contribute, e.g., $N \pi$ and $N \pi \pi$ states.

Unfortunately, our data do not allow us to determine $B_{11}$. Therefore we omit this term as well as the analogous contribution $\propto A$ in the denominator from our fit function for the ratio $R_{\mathcal{O}, \Gamma}^{\vec{p}}$. However, when performing a simultaneous fit to the ratio (44) and the two-point function (42) to extract the reduced matrix elements, the excited-state contribution $\propto A$ is taken into account in the two-point function. To fix the nucleon mass in the fits we include additional two-point correlators for $\vec{p}=\overrightarrow{0}$. The fit range of $\tau$ is restricted to the interval $2 a<\tau<t-2 a$ resulting in reasonable values of $\chi^{2} /$ d.o.f. (where d.o.f. denotes degrees of freedom). We choose on-axis momenta $\vec{p}=$ $\pm \vec{e}_{i} 2 \pi / L$ with $i$ taken to be different from the polarization direction of the nucleon, which is determined by $\Gamma$. The final analysis utilizes the data for all available momenta, nucleon polarizations and source-sink distances.

As an example we show in Fig. 1 the ratio (44) for the bare operators (25) (averaged over all members of the multiplet) with flavor $u$ on the ensemble J304. The curves represent our fit, the horizontal line and the corresponding error band show the result for the ground-state contribution.

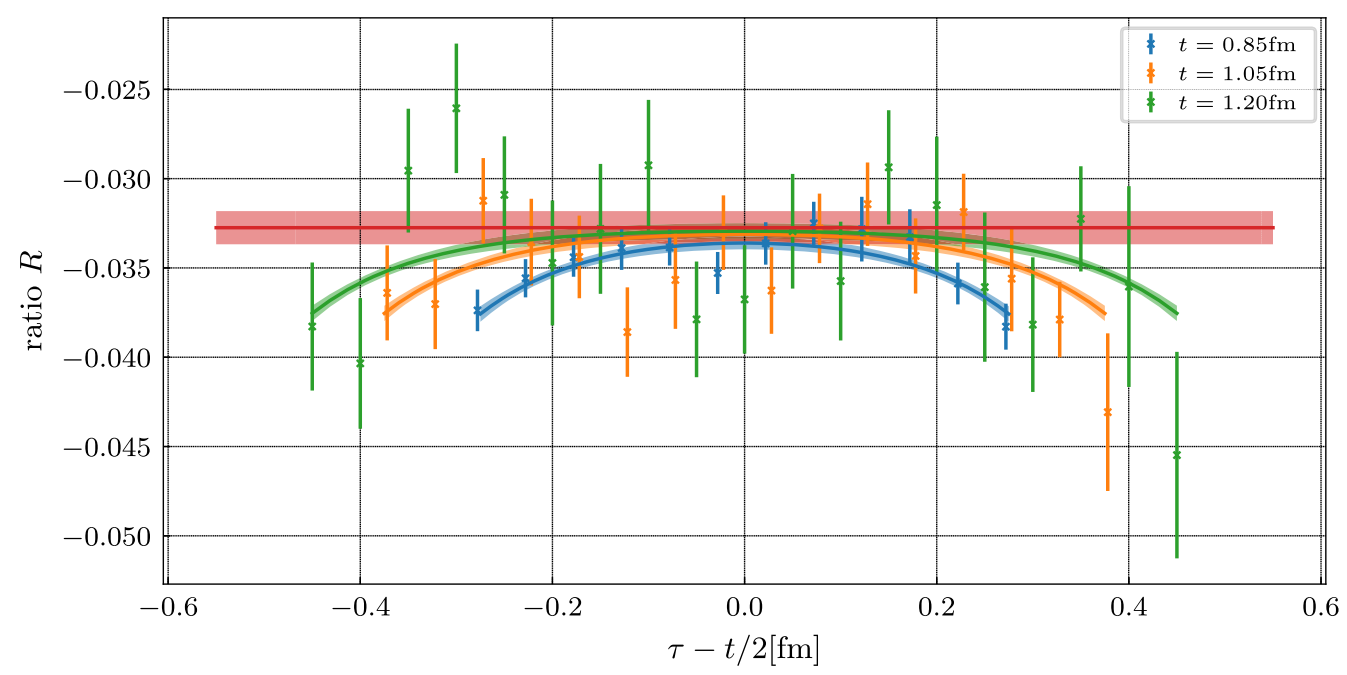

FIG. 1. The ratio $R$ of Eq. (44) for the bare operators (25) with flavor $u$ on the ensemble J304 along with our fit. The horizontal line and the corresponding error band represent the contribution of the ground state. 


\section{RESULTS}

We present our results for the light flavors $u$ and $d$ separately, where quark-line disconnected contributions have been neglected. However, we consider it to be unlikely that the latter would modify our numbers beyond the size of the other uncertainties. Superscripts $(u)$ and $(d)$ always refer to the $u$ and $d$ quarks in the proton, while $(p)$ and $(n)$ denote the matrix elements (14) for the proton and the neutron, respectively,

$$
\begin{aligned}
& d_{2}^{(p)}=\left(\frac{2}{3}\right)^{2} d_{2}^{(u)}+\left(-\frac{1}{3}\right)^{2} d_{2}^{(d)}, \\
& d_{2}^{(n)}=\left(-\frac{1}{3}\right)^{2} d_{2}^{(u)}+\left(\frac{2}{3}\right)^{2} d_{2}^{(d)} .
\end{aligned}
$$

Alternatively, one can write

$$
\begin{gathered}
d_{2}^{(p)}=\frac{3}{18} d_{2}^{(u-d)}+\frac{5}{18} d_{2}^{(u+d)}, \\
d_{2}^{(n)}=-\frac{3}{18} d_{2}^{(u-d)}+\frac{5}{18} d_{2}^{(u+d)},
\end{gathered}
$$

where $d_{2}^{(u-d)}$ does not suffer from the omission of disconnected diagrams.

Approximate renormalization in the $\mathrm{RI}^{\prime}-\mathrm{MOM}$ scheme, as described in Sec. III, is performed at some scale $\mu^{\prime}$. We attempt a combined continuum and chiral extrapolation using the fit functions (53)-(55) given below and evolve the results to the scale $\mu=2 \mathrm{GeV}$ with the help of the one-loop formula for the flavor-nonsinglet operators,

$$
d_{2}(\mu)=\left(\frac{\alpha_{s}\left(\mu^{\prime}\right)}{\alpha_{s}(\mu)}\right)^{-B} d_{2}\left(\mu^{\prime}\right),
$$

where

$$
B=\frac{1}{\frac{11}{3} N_{c}-\frac{2}{3} N_{f}}\left(3 N_{c}-\frac{1}{6}\left(N_{c}-\frac{1}{N_{c}}\right)\right)
$$

with $N_{c}=3$ and $N_{f}=3$. As we neglect disconnected contributions, we use this value of $B$, which is strictly speaking only correct for the $(u-d)$ part, also for $(u+d)$. Varying the intermediate scale $\mu^{\prime}$ should give us some measure of the uncertainty related to the renormalization. The central results, however, are all obtained at $\mu^{\prime}=\mu$, so they do not depend on the perturbative value of $B$.

When constructing our fit functions we take into account that the leading discretization effects in the matrix elements in our simulations are $O(a)$. For the continuum and chiral extrapolation of the $d_{2}$ and $a_{2}$ data, we consider the fit formulas

$$
f_{1}\left(a, m_{\pi}, m_{K}\right)=C_{1}+C_{2} a+C_{3} \delta m^{2}+C_{4} \bar{m}^{2},
$$

$$
\begin{gathered}
f_{2}\left(a, m_{\pi}, m_{K}\right)=C_{1}+C_{2} a+C_{3} \delta m^{2}+C_{4} \bar{m}^{2}+C_{5} a^{2}, \\
f_{3}\left(a, m_{\pi}, m_{K}\right)= \\
C_{1}+C_{2} a+C_{3} \delta m^{2}+C_{4} \bar{m}^{2} \\
+C_{5} \bar{m}^{2} a+C_{6} \delta m^{2} a,
\end{gathered}
$$

where

$$
\delta m^{2}=m_{K}^{2}-m_{\pi}^{2}, \quad \bar{m}^{2}=\left(2 m_{K}^{2}+m_{\pi}^{2}\right) / 3 .
$$

The difference between the corresponding results should provide an impression of the uncertainty inherent in the fit procedure. The gauge field ensembles used in the fits are collected in Table I. Note that on a few configurations in some of the coarser ensembles (H105, H106, H107, N450, B452 and N201) we encountered measurements that were separated by more than a hundred $68 \%$ confidence level intervals from the results obtained on the remaining configurations. The origin of these deviations

TABLE II. Results for $d_{2}$ at $\mu=2 \mathrm{GeV}$ obtained with the renormalization $\tilde{Z}$ and the extrapolation functions (53)-(55). Superscripts $(u)$ and $(d)$ refer to the $u$ and $d$ quarks in the proton.

\begin{tabular}{cccccccc}
\hline \hline$\mu^{\prime 2}\left(\mathrm{GeV}^{2}\right)$ & Fit form & $d_{2}^{(u)}(\mu)$ & $d_{2}^{(d)}(\mu)$ & $d_{2}^{(u-d)}(\mu)$ & $d_{2}^{(u+d)}(\mu)$ & $d_{2}^{(p)}(\mu)$ & $d_{2}^{(n)}(\mu)$ \\
\hline 4.0 & $f_{1}$ & $0.026(4)$ & $-0.0086(26)$ & $0.034(4)$ & $0.018(5)$ & $0.0105(19)$ & $-0.0009(14)$ \\
8.0 & $f_{1}$ & $0.024(4)$ & $-0.0090(29)$ & $0.033(4)$ & $0.016(6)$ & $0.0098(21)$ & $-0.0012(15)$ \\
12.0 & $f_{1}$ & $0.024(5)$ & $-0.0092(31)$ & $0.033(5)$ & $0.015(6)$ & $0.0095(22)$ & $-0.0013(16)$ \\
4.0 & $f_{2}$ & $0.028(14)$ & $0.006(9)$ & $0.023(14)$ & $0.036(19)$ & $0.013(7)$ & $0.006(5)$ \\
4.0 & $f_{3}$ & $0.039(13)$ & $0.001(9)$ & $0.036(13)$ & $0.040(18)$ & $0.017(6)$ & $0.005(5)$ \\
\hline \hline
\end{tabular}



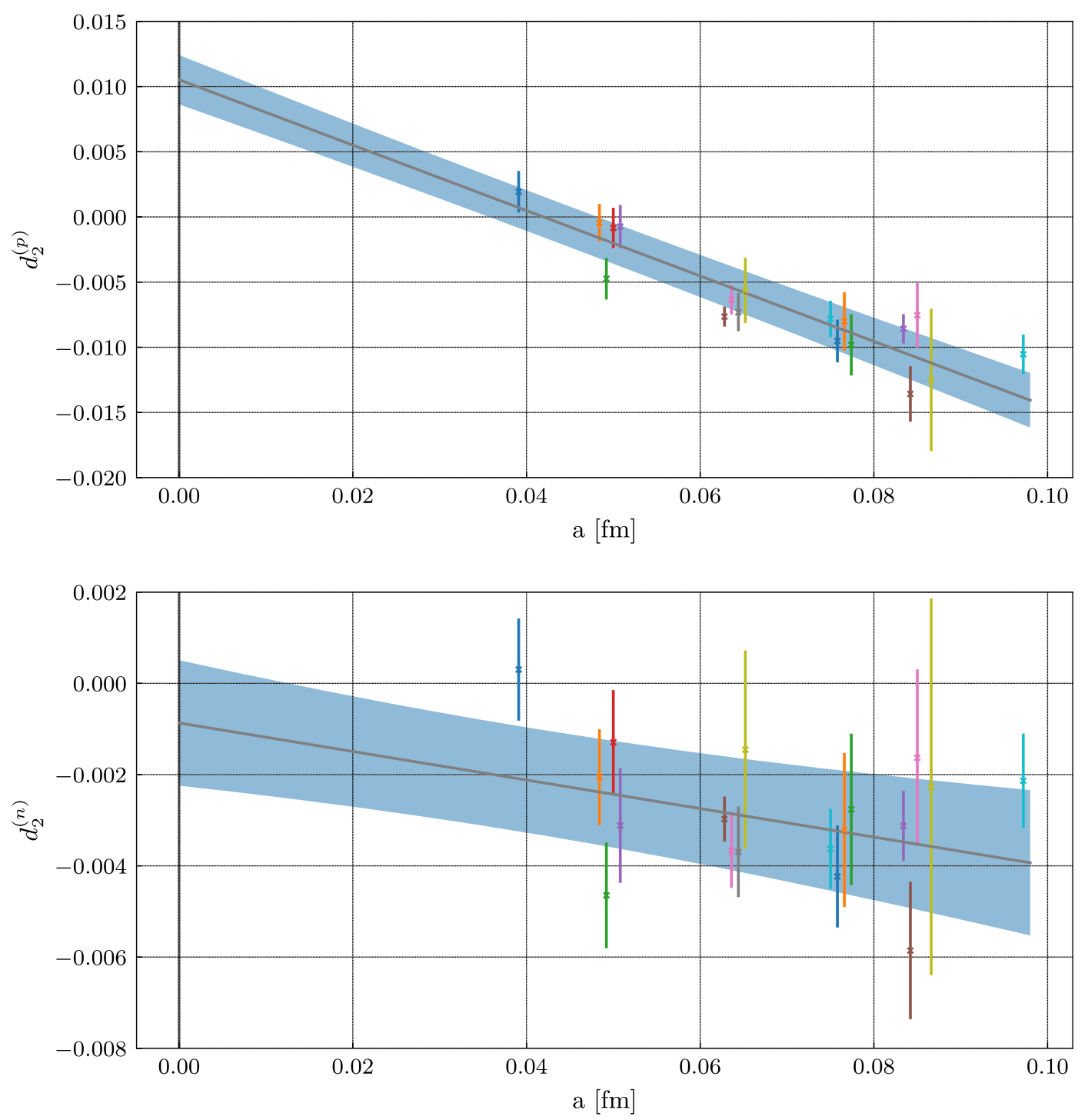

FIG. 2. Continuum extrapolation of $d_{2}^{(p)}$ and $d_{2}^{(n)}$. This extrapolation corresponds to the first line of Table II. Results belonging to the same value of $a$ are horizontally shifted for visibility by a small amount such that the exact value of $a$ lies in the center of the respective group. Within each of these groups the pion mass decreases from left to right. The results from the ensembles A654, H105 and D451 are not shown on the plots because of their large error bars.

\begin{tabular}{|c|c|c|c|c|}
\hline J501 & $\neq$ & N203 & \$ & H101 \\
\hline N300 & $\neq$ & N201 & & H107 \\
\hline N304 & 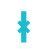 & B450 & & $\mathrm{H} 102$ \\
\hline N302 & & S400 & & H105 \\
\hline J304 & & B 452 & & H106 \\
\hline N202 & & N450 & & A653 \\
\hline N204 & & D451 & & A654 \\
\hline
\end{tabular}

FIG. 3. Legend for Figs. 2, 4 and 5. The order of the ensembles (from top left to bottom right) is the same as in these figures (from left to right). is unclear and we excluded these configurations from further analysis.

The fit results for $d_{2}$ are given in Table II for the case of the renormalization matrix $\tilde{Z}$, cf. Sec. III. The dependence on the intermediate scale $\mu^{\prime}$ is quite weak, due to the use of $\tilde{Z}$. However, the dependence on the choice of the fit function is more pronounced, although all three functions yield reasonable fits with $\chi^{2} /$ d.o.f. between 0.66 and 0.94. In Fig. 2 we plot our data along with the fit function (53) for $\mu^{\prime}=2 \mathrm{GeV}$ versus the lattice spacing $a$. The legend identifying the ensembles is 


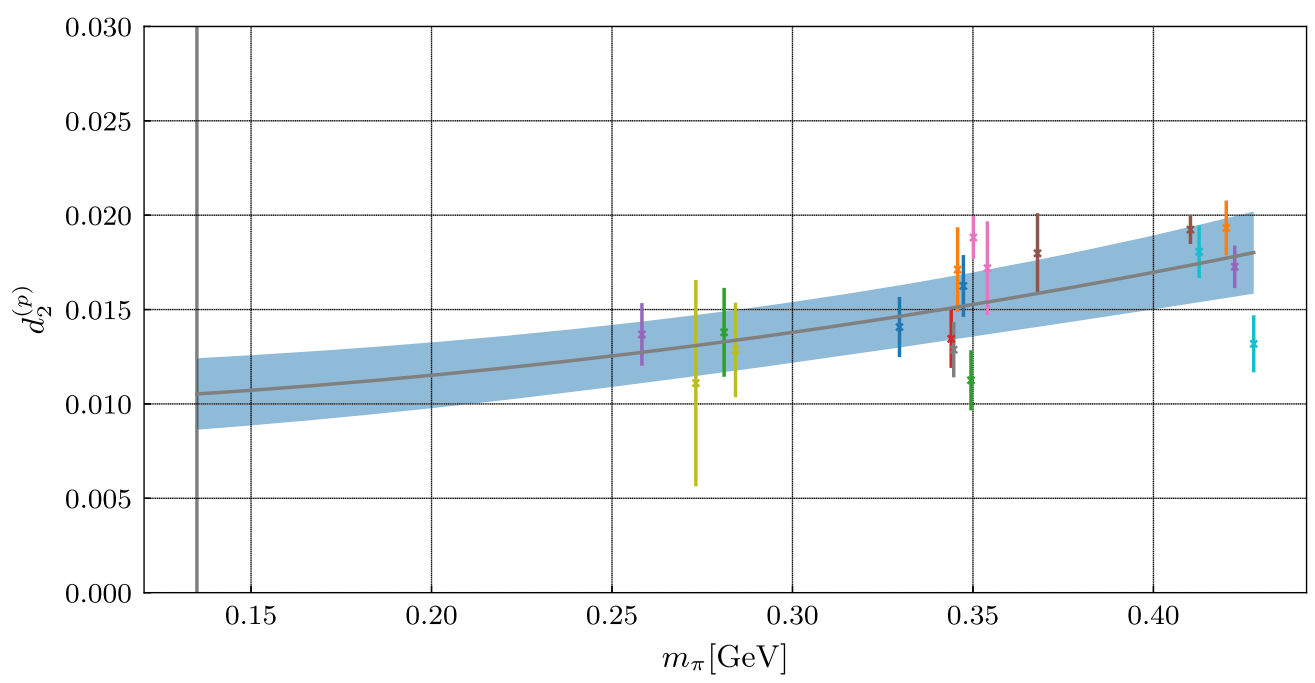

FIG. 4. Mass dependence of $d_{2}^{(p)}$. The fit curve corresponds to the first line of Table II. The results from the ensembles A654, H105 and D451 are not shown on the plot because of their large error bars.

given in Fig. 3. The curve shows the fit function evaluated with the physical values of $m_{\pi}$ and $m_{K}$. The fitted coefficients $C_{3}$ and $C_{4}$ have been used to shift the data points vertically such that they correspond to the physical masses.

In order to visualize the mass dependence we show in Fig. 4 the results for $d_{2}^{(p)}$ together with the fit corresponding to the first line of Table II plotted against $m_{\pi}$. The curve represents the function $f_{1}\left(0, m_{\pi}, m_{K}^{\text {phys }}\right)$ evaluated with the fitted parameters, and the data points have been shifted by subtracting $f_{1}\left(a, m_{\pi}, m_{K}\right)-f_{1}\left(0, m_{\pi}, m_{K}^{\text {phys }}\right)$. Compared to the $a$ dependence, the dependence on $m_{\pi}$ turns out to be more moderate.

Results for the twist- 2 matrix element $a_{2}$ are presented in Table III. The flavor dependence is of the same form as in the case of $d_{2}$. Since the operators that we use for the determination of $a_{2}$ are multiplicatively renormalizable, we can follow the standard $\mathrm{RI}^{\prime}$-MOM procedure to obtain values in the $\overline{\mathrm{MS}}$ scheme at $\mu=2 \mathrm{GeV}$. As in the case of $d_{2}$, we obtain reasonable fits with all three fit functions $\left(0.80 \leq \chi^{2} /\right.$ d.o.f. $\left.\leq 0.92\right)$, but find some dependence of the results for $a_{2}$ on the fit function. Plots of our $a_{2}$ data and the fit function (53) are shown in Fig. 5, which is analogous to Fig. 2. Again, the dependence on the pion mass appears to be rather weak.

For our final values, collected in Table IV, we take the results given in the first line of Table II for $d_{2}$ and Table III for $a_{2}$. The errors in these tables are purely statistical, but there are several sources of systematic uncertainties. Comparing the results obtained by varying the fit function or the scale $\mu^{\prime}$ allows us to estimate the influence of the extrapolation method. Since the renormalization in the case of $a_{2}$ is less subtle than in the case of $d_{2}$, we have refrained from varying the intermediate renormalization scale $\mu^{\prime}$ in the analysis for $a_{2}$. As the estimate of the systematic error due to our extrapolation we take the maximum of the (absolute value of the) difference between the final value and the results obtained by means of the fit functions $f_{2}$ and $f_{3}$.

TABLE III. Results for $a_{2}$ in the $\overline{\mathrm{MS}}$ scheme at $\mu=2 \mathrm{GeV}$ obtained with the extrapolation functions (53)-(55). Superscripts $(u)$ and $(d)$ refer to the $u$ and $d$ quarks in the proton.

\begin{tabular}{lcccclcr}
\hline \hline$\mu^{\prime 2}\left(\mathrm{GeV}^{2}\right)$ & Fit form & $a_{2}^{(u)}(\mu)$ & $a_{2}^{(d)}(\mu)$ & $a_{2}^{(u-d)}(\mu)$ & $a_{2}^{(u+d)}(\mu)$ & $a_{2}^{(p)}(\mu)$ & $a_{2}^{(n)}(\mu)$ \\
\hline 4.0 & $f_{1}$ & $0.161(11)$ & $-0.025(6)$ & $0.187(10)$ & $0.136(14)$ & $0.069(5)$ & $0.0068(33)$ \\
4.0 & $f_{2}$ & $0.195(35)$ & $-0.016(21)$ & $0.208(35)$ & $0.18(5)$ & $0.085(17)$ & $0.015(11)$ \\
4.0 & $f_{3}$ & $0.137(33)$ & $-0.038(19)$ & $0.175(31)$ & $0.10(4)$ & $0.057(15)$ & $-0.001(10)$ \\
\hline \hline
\end{tabular}



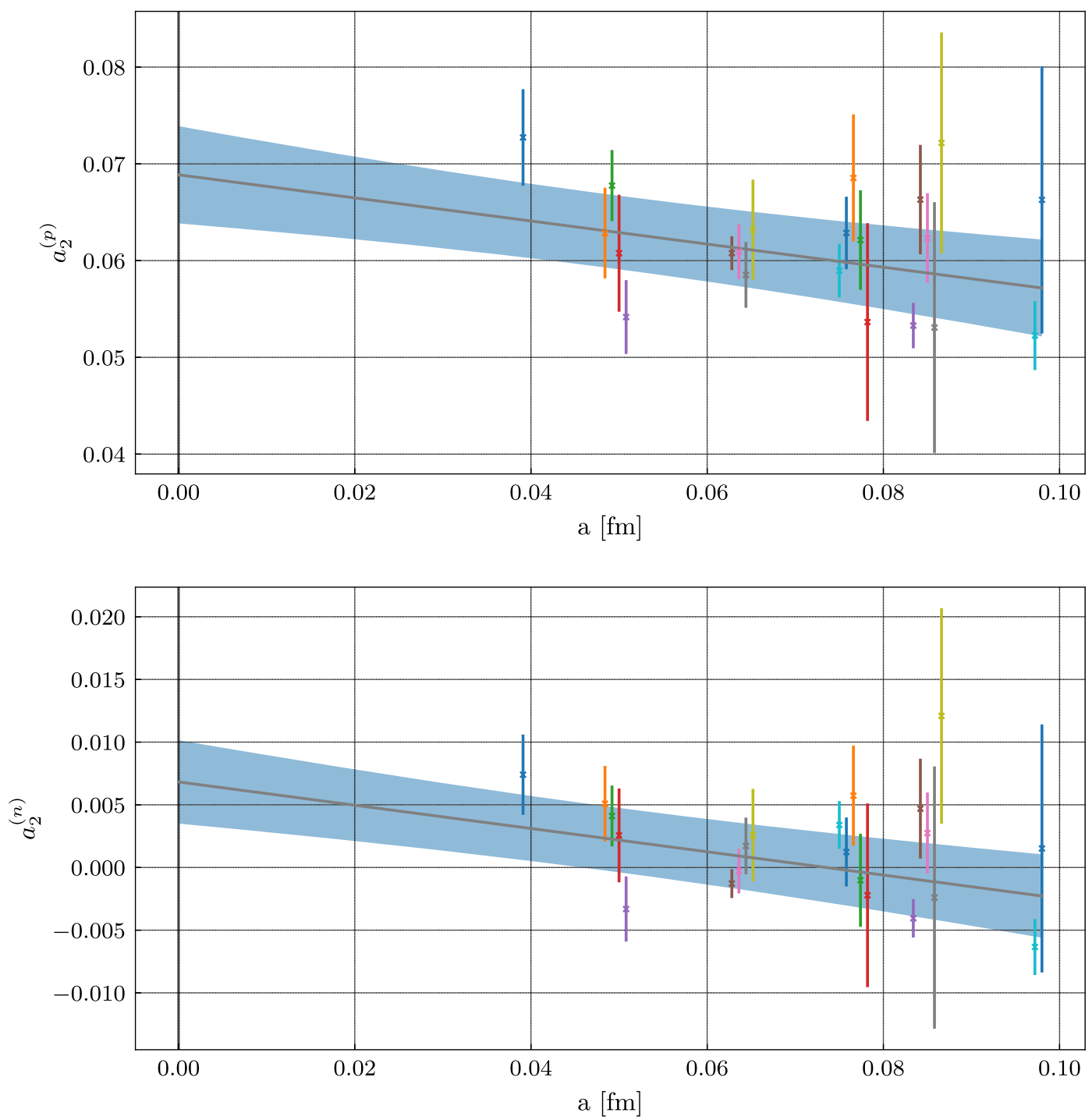

FIG. 5. Continuum extrapolation of $a_{2}^{(p)}$ and $a_{2}^{(n)}$. This extrapolation corresponds to the first line of Table III. Results belonging to the same value of $a$ are horizontally shifted for visibility by a small amount such that the exact value of $a$ lies in the center of the respective group. Within each of these groups the pion mass decreases from left to right.

Unfortunately, we are not able to assess the effect of neglecting the operators (27) in the process of the renormalization of $d_{2}$. This problem must be left for future investigations. Another source of error that cannot yet be quantified is the omission of quark-line disconnected contributions, which leaves only $a_{2}^{(u-d)}$ and $d_{2}^{(u-d)}$ unaffected. However, we expect that this error is small compared to the other uncertainties.

TABLE IV. Final results for $a_{2}$ ( $\overline{\mathrm{MS}}$ scheme) and for $d_{2}$ (renormalization $\tilde{Z}$ ), both at $\mu=2 \mathrm{GeV}$. The first error is statistical, while the second error accounts for the uncertainty due to the combined chiral and continuum extrapolations. The error caused by the approximations in the renormalization procedure for $d_{2}$ cannot be quantified.

\begin{tabular}{lllllll}
\hline \hline & \multicolumn{1}{c}{$\square_{2}^{(u)}(\mu)$} & \multicolumn{1}{c}{$\square_{2}^{(d)}(\mu)$} & $\square_{2}^{(u-d)}(\mu)$ & $\square_{2}^{(u+d)}(\mu)$ & $\square_{2}^{(p)}(\mu)$ & $\square_{2}^{(n)}(\mu)$ \\
\hline$\square=d$ & $0.026(4)(13)$ & $-0.0086(26)(146)$ & $0.034(4)(11)$ & $0.018(5)(22)$ & $0.0105(19)(65)$ & $-0.0009(14)(69)$ \\
$\square=a$ & $0.161(11)(34)$ & $-0.025(6)(13)$ & $0.187(10)(21)$ & $0.136(14)(44)$ & $0.069(5)(16)$ & $0.0068(33)(82)$ \\
\hline \hline
\end{tabular}




\section{COMPARISON WITH EXPERIMENT}

Several experiments have measured the structure functions $g_{i}\left(x, Q^{2}\right)$ for certain ranges of the variables $x$ and $Q^{2}$ and attempted to determine the moments

$$
\int_{0}^{1} d x x^{2} g_{i}\left(x, Q^{2}\right)
$$

for the proton as well as for the neutron. Results are given for $Q^{2}$ up to $5 \mathrm{GeV}^{2}$. As far as we can see, the Wilson coefficients are taken into account only in leading order. In this approximation the moments of the structure functions are related to the reduced matrix elements by

$$
a_{2}(\mu)=4 \int_{0}^{1} d x x^{2} g_{1}\left(x, Q^{2}\right)
$$

and

$$
d_{2}(\mu)=2 \int_{0}^{1} d x x^{2}\left(2 g_{1}\left(x, Q^{2}\right)+3 g_{2}\left(x, Q^{2}\right)\right)
$$

with $\mu^{2}=Q^{2}$.

Let us begin with the results for $a_{2}$. In Table $\mathrm{V}$ we collect the values given for $\int_{0}^{1} d x x^{2} g_{1}\left(x, Q^{2}\right)$ in the literature with statistical and systematic errors (if they are given separately) added in quadrature.

In order to compare the results given in Table $\mathrm{V}$ with our numbers we take into account the one-loop QCD corrections, which are flavor independent, i.e., we divide the experimental values of $\int_{0}^{1} d x x^{2} g_{1}\left(x, Q^{2}\right)$ by the Wilson coefficient (9) with $\mu^{2}=Q^{2}$. Then we use the renormalization group to evolve the renormalization scale of the resulting matrix element $a_{2}$ to our value $\mu^{2}=4 \mathrm{GeV}^{2}$. We employ the five-loop anomalous dimension of the relevant operator multiplet along with the five-loop $\beta$ function. The details of the calculation are the same as in Ref. [42]. The resulting values for $a_{2}(2 \mathrm{GeV})$ can be found in Table VI.

In Table VII we collect the values presented for $d_{2}^{\text {alt }}=$ $d_{2} / 2$ in the literature with statistical and systematic errors (if they are given separately) added in quadrature. As in this case Wilson coefficients beyond tree level are not available, we just use the renormalization group to evolve the renormalization scale to our value $\mu^{2}=4 \mathrm{GeV}^{2}$. The corresponding factors are calculated from Eqs. (51) and

TABLE V. Experimental results for $\int_{0}^{1} d x x^{2} g_{1}\left(x, Q^{2}\right)$.

\begin{tabular}{lclc}
\hline \hline References & $Q^{2}\left(\mathrm{GeV}^{2}\right)$ & \multicolumn{1}{c}{ Proton } & Neutron \\
\hline$[13]$ & 5.0 & $0.0124(10)$ & $-0.0024(16)$ \\
{$[62]$} & 4.2 & $0.01100(83)$ & $\ldots$ \\
{$[62]$} & 5.0 & $0.00853(175)$ & $\ldots$ \\
{$[18]$} & 3.21 & $\ldots$ & $0.00086(64)$ \\
{$[18]$} & 4.32 & $\ldots$ & $0.00050(65)$ \\
\hline \hline
\end{tabular}

TABLE VI. Values for $a_{2}$ with $\mu^{2}=4 \mathrm{GeV}^{2}$ from experiment.

\begin{tabular}{lccc}
\hline \hline References & $Q^{2}\left(\mathrm{GeV}^{2}\right)$ & $a_{2}^{(p)}(\mu)$ & $a_{2}^{(n)}(\mu)$ \\
\hline$[13]$ & 5.0 & $0.0497(40)$ & $-0.0096(64)$ \\
{$[62]$} & 4.2 & $0.0427(32)$ & $\ldots$ \\
{$[62]$} & 5.0 & $0.0342(70)$ & $\ldots$ \\
{$[18]$} & 3.21 & $\ldots$ & $0.0032(24)$ \\
{$[18]$} & 4.32 & $\ldots$ & $0.0020(25)$ \\
\hline \hline
\end{tabular}

(52), and the resulting values for $d_{2}(2 \mathrm{GeV})$ can be found in Table VIII.

As in the analysis of the experimental data the variation of $Q^{2}$ in the respective experimental setup generally was not taken into account, applying the renormalization group running is not too well justified, but the effect is anyhow quite small compared to the experimental uncertainties. If, however, the sign change from negative numbers at the smaller values of $Q^{2}$ to positive numbers at larger $Q^{2}$ is taken seriously and interpreted as a "nontrivial scale dependence" [19], the perturbative renormalization group would not be applicable in this range of $Q^{2}$.

In addition to these results from single experiments, there is also a global analysis of polarized inclusive deepinelastic scattering available [63]. Unfortunately, the resulting values for $d_{2}^{(p)}$ and $d_{2}^{(n)}$ are given at the rather low scale $Q^{2}=1 \mathrm{GeV}^{2}$. If one nevertheless uses Eq. (51) for the evolution to the scale $4 \mathrm{GeV}^{2}$, one finds $d_{2}^{(p)}(2 \mathrm{GeV})=$ $0.0062(25)$ and $d_{2}^{(n)}(2 \mathrm{GeV})=-0.0012(12)$ in broad agreement with the individual results in Table VIII.

Our results for $a_{2}^{(p)}$ in Table III are larger than the experimental values, but the dependence on the choice of the fit function may indicate that this discrepancy should not be taken too seriously. A similar tendency is observed in Fig. 20 of Ref. [64], where moments obtained from quasidistributions are compared with phenomenological determinations. Figure 6 of Ref. [65] (see also Ref. [60]) seems to suggest that a more sophisticated chiral

TABLE VII. Experimental results for $d_{2}^{\text {alt }}=d_{2} / 2$. In the case of Ref. [12] we have chosen the "SLAC average."

\begin{tabular}{lccc}
\hline \hline References & $Q^{2}\left(\mathrm{GeV}^{2}\right)$ & $d_{2}^{(p)} / 2$ & $d_{2}^{(n)} / 2$ \\
\hline$[12]$ & 3.0 & $\ldots$ & $-0.010(15)$ \\
{$[13]$} & 5.0 & $0.0058(50)$ & $0.0050(210)$ \\
{$[14]$} & 5.0 & $0.0032(17)$ & $0.0079(48)$ \\
{$[15]$} & 5.0 & $\ldots$ & $0.0062(28)$ \\
{$[62]$} & 4.2 & $0.0014(130)$ & $\ldots$ \\
{$[62]$} & 5.0 & $0.0035(150)$ & $\ldots$ \\
{$[16]$} & 5.0 & $0.0148(107)$ & $\ldots$ \\
{$[17]$} & 3.21 & $\ldots$ & $-0.00421(114)$ \\
{$[17]$} & 4.32 & $\ldots$ & $-0.00035(108)$ \\
{$[19]$} & 2.8 & $-0.00414(328)$ & $\ldots$ \\
{$[19]$} & 4.3 & $-0.00149(400)$ & $\ldots$ \\
\hline \hline
\end{tabular}


TABLE VIII. Results for $d_{2}$ with $\mu^{2}=4 \mathrm{GeV}^{2}$ from experiment. In the case of Ref. [12] we have chosen the "SLAC average".

\begin{tabular}{lccc}
\hline \hline References & $Q^{2}\left(\mathrm{GeV}^{2}\right)$ & $d_{2}^{(p)}(\mu)$ & $d_{2}^{(n)}(\mu)$ \\
\hline$[12]$ & 3.0 & $\ldots$ & $-0.019(28)$ \\
{$[13]$} & 5.0 & $0.0122(106)$ & $0.0106(443)$ \\
{$[14]$} & 5.0 & $0.0068(36)$ & $0.0167(101)$ \\
{$[15]$} & 5.0 & $\ldots$ & $0.0131(59)$ \\
{$[62]$} & 4.2 & $0.0028(263)$ & $\ldots$ \\
{$[62]$} & 5.0 & $0.0074(317)$ & $\ldots$ \\
{$[16]$} & 5.0 & $0.0312(226)$ & $\ldots$ \\
{$[17]$} & 3.21 & $\ldots$ & $-0.0080(22)$ \\
{$[17]$} & 4.32 & $\ldots$ & $-0.0007(22)$ \\
{$[19]$} & 2.8 & $-0.0075(60)$ & $\ldots$ \\
{$[19]$} & 4.3 & $-0.0030(81)$ & $\ldots$ \\
\hline \hline
\end{tabular}

extrapolation could diminish this discrepancy, but better data are needed to clarify this issue. Concerning $a_{2}^{(n)}$ we can hardly say more than that it must be quite small, as also indicated by the experimental values.

The experimental results for $d_{2}^{(p)}$ collected in Table VIII may perhaps be summarized in the statement that the data taken at reasonably large $Q^{2}$ hint at a value in the vicinity of 0.01 for $\mu=2 \mathrm{GeV}$. The results for $d_{2}^{(n)}$ are not so easy to summarize. While the order of magnitude is 0.01 as well, even the sign is ambiguous. For the final numbers from our lattice calculation see Table IV. We find a value for $d_{2}^{(n)}$ which is consistent with zero. For $d_{2}^{(p)}$ we get a number quite close to 0.01 , which is consistent with most of the experimental determinations.

\section{COMPARISON WITH OTHER LATTICE DETERMINATIONS}

There are a few previous lattice investigations of $a_{2}$ and $d_{2}$, with which we can compare our new results. For this purpose we consider the reduced matrix elements $a_{2}^{(q)}$ and $d_{2}^{(q)}$ with $q=u, d$.

In Ref. [11] a continuum limit was not attempted. Instead, the results on the finest lattice in the chiral limit were considered as the best estimates obtained in this $N_{f}=$ 2 simulation. With the help of the perturbative running factor we evolve our $a_{2}$ results from $\mu^{2}=4 \mathrm{GeV}^{2}$ to the scale $\mu^{2}=5 \mathrm{GeV}^{2}$ used in Ref. [11]. This yields $a_{2}^{(u)}=$ $0.155(11)(33)$ and $a_{2}^{(d)}=-0.024(6)(13)$ to be compared with $a_{2}^{(u)}=0.187(28)$ and $a_{2}^{(d)}=-0.056(11)$. In the case of $d_{2}$ we obtain $d_{2}^{(u)}=0.025(4)(12)$ and $d_{2}^{(d)}=$ $-0.0081(25)(138)$ at $\mu^{2}=5 \mathrm{GeV}^{2}$ to be compared with the values $d_{2}^{(u)}=0.010(12)$ and $d_{2}^{(d)}=-0.0056(50)$ given in Ref. [11]. The statistical errors of our present determination are significantly smaller than those quoted in
Ref. [11], while the central values are in rough agreement with each other. Unfortunately, the uncertainties due to the combined chiral and continuum extrapolations, which could not be estimated in the previous study, are still rather large.

Values for $\left\langle x^{2}\right\rangle_{\Delta u}=a_{2}^{(u)} / 2$ and $\left\langle x^{2}\right\rangle_{\Delta d}=a_{2}^{(d)} / 2$ from another $N_{f}=2$ simulation are presented in Ref. [66]. They are obtained at a single lattice spacing $a \approx 0.1 \mathrm{fm}$ with the help of perturbative renormalization. Since the results are given at $\mu^{2}=4 \mathrm{GeV}^{2}$ in the $\overline{\mathrm{MS}}$ scheme, they can directly be compared with our numbers. From Table IX of Ref. [66] we get $a_{2}^{(u)}=0.232(84)$ and $a_{2}^{(d)}=0.002(50)$. Although not extrapolated to the continuum, these values are roughly compatible with our results.

Somewhat indirect information on $a_{2}^{(u-d)}$ is contained in Ref. [60]. This paper relies on simulations with $N_{f}=2+1$ flavors at a single lattice spacing $a=0.124 \mathrm{fm}$ and employs a combination of perturbative and nonperturbative methods for the renormalization. Results are given at a renormalization scale $\mu^{2} \approx 2.5 \mathrm{GeV}^{2}$ for the generalized form factors $\tilde{A}_{10}^{u-d}(t)$ and $\tilde{A}_{30}^{u-d}(t)$, which are related to $\left\langle x^{2}\right\rangle_{\Delta u-\Delta d}=a_{2}^{(u-d)} / 2$ through

$$
\left\langle x^{2}\right\rangle_{\Delta u-\Delta d}=\tilde{A}_{30}^{u-d}(0)=\left.\frac{\tilde{A}_{30}^{u-d}(t)}{\tilde{A}_{10}^{u-d}(t)}\right|_{t=0} \tilde{A}_{10}^{u-d}(0)
$$

with $\tilde{A}_{10}^{u-d}(0)=g_{A}$. Reading off from Fig. 32 in Ref. [60] the value

$$
\left.\frac{\tilde{A}_{30}^{u-d}(t)}{\tilde{A}_{10}^{u-d}(t)}\right|_{t=0} \approx 0.09
$$

and using $g_{A} \approx 1.27$ one obtains $a_{2}^{(u-d)} \approx 0.229$ for $\mu^{2} \approx 2.5 \mathrm{GeV}^{2}$. This value corresponds to $a_{2}^{(u-d)} \approx 0.209$ at $\mu^{2}=4 \mathrm{GeV}^{2}$, consistent with our result in Table IV.

Results from the quasidistribution approach [67] lead to the conclusion that the Wandzura-Wilczek approximation for $g_{T}(x)=g_{1}(x)+g_{2}(x)$ works well at least up to $x \approx 0.4$. Whether the deviations observed at higher values of $x$ indicate nonvanishing twist-3 effects or have a different origin remains to be seen.

\section{CONCLUSIONS}

In the present paper we computed the nucleon matrix elements $a_{2}$ and $d_{2}$, which determine the $x^{2}$ moments of the spin structure functions $g_{1}$ and $g_{2}$, in lattice QCD, thus improving on the earlier evaluation [11] (coauthored by some of us). In both determinations quark-line disconnected contributions were neglected.

For the twist-2 matrix element $a_{2}$ we found $a_{2}^{(p)}=0.069(5)(16)$ and $a_{2}^{(n)}=0.0068(33)(82)$ at the 


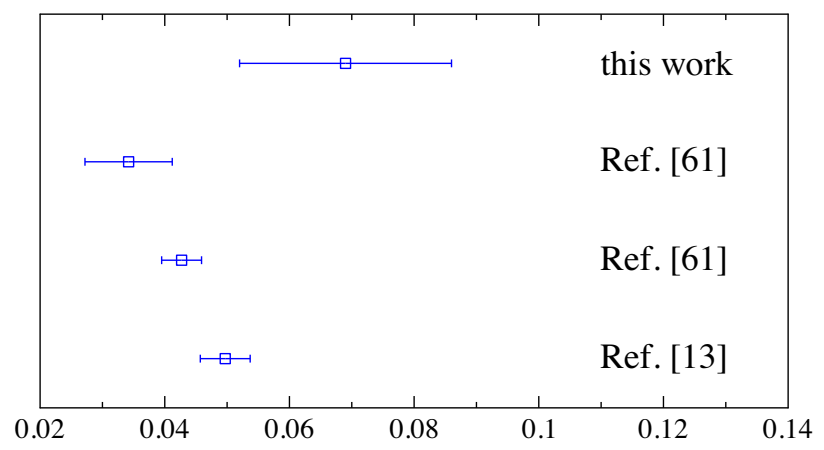

FIG. 6. Comparison of our results for $a_{2}^{(p)}$ with experimental values. The renormalization scale is $\mu^{2}=4 \mathrm{GeV}^{2}$. Statistical and systematic errors have been added in quadrature.

renormalization scale $\mu=2 \mathrm{GeV}$, see also Table IV. In both cases the second (systematic) error is considerably larger than the first (statistical) error. Our results are in broad agreement with phenomenology, as shown in Figs. 6 and 7.

The parameter $d_{2}$ quantifies a specific twist-3 quarkgluon correlation in the nucleon. Because it is experimentally accessible it became a much discussed test case for our understanding of hadron structure beyond twist 2 . We observed a strong dependence on lattice spacing for $d_{2}^{(p)}$, see Fig. 2, which implies that the good agreement between the earlier lattice result and experiment probably was somewhat accidental. In contrast, in this new lattice determination of $d_{2}^{(p)}$ and $d_{2}^{(n)}$ we take the lattice spacing dependence into account and only when doing this, the results agree well with experiment (and, therefore, also with the numbers given in Ref. [11]). Note also that the $a$ dependence of $d_{2}^{(n)}$ is far less pronounced. These results provide a showcase example justifying the CLS strategy to focus its resources on controlling the continuum limit. The $a$ dependence of any observable of interest can be strong (as for $d_{2}^{(p)}$ ) or weak (as for $d_{2}^{(n)}$ ). What is the case has to be carefully evaluated for each specific quantity.

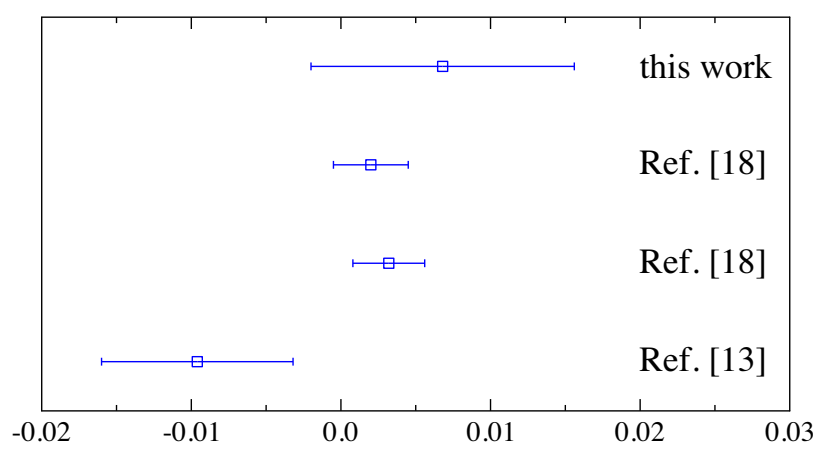

FIG. 7. Comparison of our results for $a_{2}^{(n)}$ with experimental values. The renormalization scale is $\mu^{2}=4 \mathrm{GeV}^{2}$. Statistical and systematic errors have been added in quadrature.

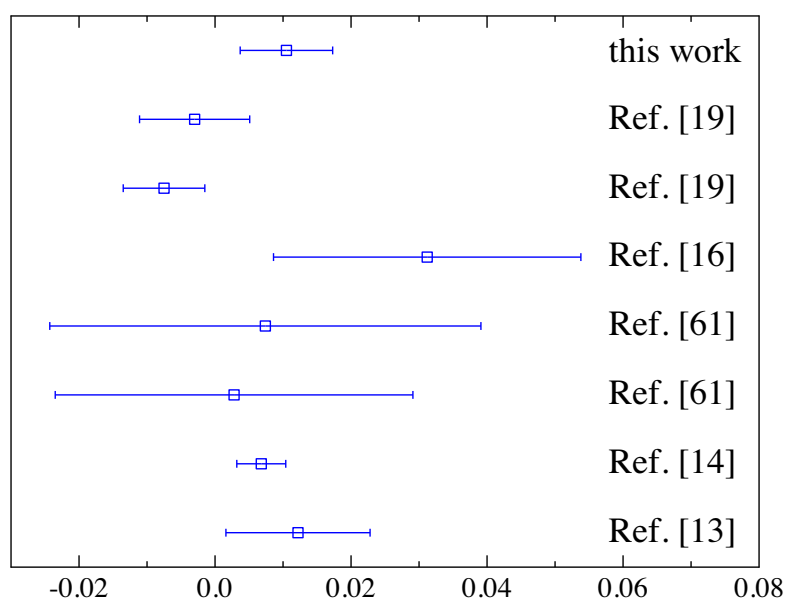

FIG. 8. Comparison of our results for $d_{2}^{(p)}$ with experimental values. The renormalization scale is $\mu^{2}=4 \mathrm{GeV}^{2}$. Statistical and systematic errors have been added in quadrature.

The final results can be found in Table IV. We obtained $d_{2}^{(p)}=0.0105(19)(65)$ and $d_{2}^{(n)}=-0.0009(14)(69)$ at the renormalization scale $\mu=2 \mathrm{GeV}$. Again, the systematic error dominates the total one. In Figs. 8 and 9 we compare our findings with results from the experimental and phenomenological literature.

Following Refs. $[9,10]$ these numbers can be related to the transverse color Lorentz force on a quark in a transversely polarized proton. Considering the proton in its rest frame with $p^{+}=m_{N} / \sqrt{2}$ and $s^{x}=m_{N}$, one gets in analogy to Eq. (1)

$$
F^{q, y}(0)=m_{N}^{2} d_{2}^{(q)}
$$

With $m_{N}^{2} \approx 4.47 \mathrm{GeV} / \mathrm{fm}$ we obtain

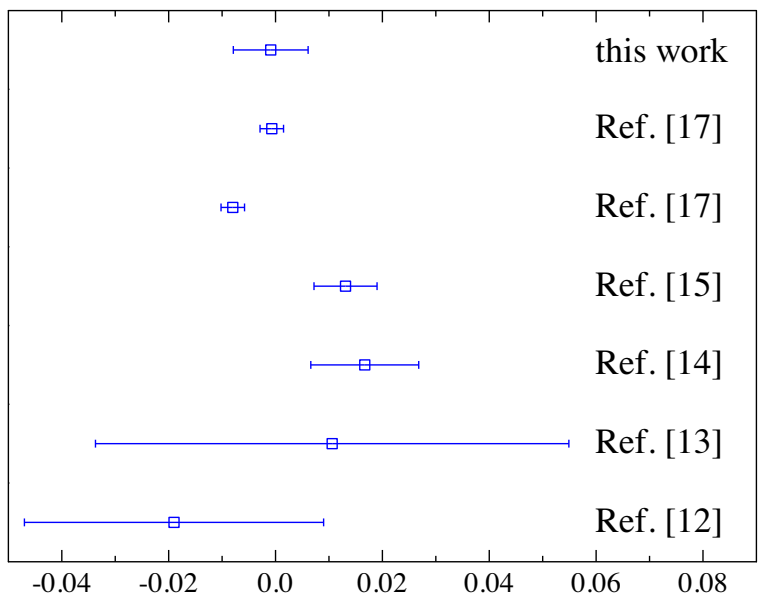

FIG. 9. Comparison of our results for $d_{2}^{(n)}$ with experimental values. The renormalization scale is $\mu^{2}=4 \mathrm{GeV}^{2}$. Statistical and systematic errors have been added in quadrature. 


$$
\begin{aligned}
& F^{u, y}(0)=116(61) \mathrm{MeV} / \mathrm{fm}, \\
& F^{d, y}(0)=-38(66) \mathrm{MeV} / \mathrm{fm},
\end{aligned}
$$

where we have added the two errors in quadrature.

\section{ACKNOWLEDGMENTS}

The authors thank V. M. Braun, W. Söldner and S. Weishäupl for discussions and valuable input and our colleagues in the Coordinated Lattice Simulations effort (CLS $[44,68]$ ) for the joint generation of the gauge field ensembles. We used a modified version of the CHROMA [61] software package, along with improved linear solvers $[45,52,53,69]$. The gauge ensembles were generated as part of the CLS effort, using OpenQCD [45,70]. This work was supported by the DFG (Deutsche Forschungsgemeinschaft) through the collaborative research center SFB/TRR-55 and the Research Unit FOR 2926 "Next Generation $\mathrm{pQCD}$ for Hadron Structure: Preparing for the EIC" and the European Union's Horizon 2020 research and innovation program under the Marie Skłodowska-Curie Grant Agreement No. 813942 (ITN EuroPLEx) and No. 824093 (STRONG-2020). A. Sternbeck acknowledges support by the BMBF (German Federal Ministry of Education and Research) under Grant No. 05P15SJFAA (FAIR-APPA-SPARC) and by the DFG Research Training Group GRK1523. The authors gratefully acknowledge computing time granted by the John von Neumann Institute for Computing (NIC) and provided on the Booster partition of the supercomputer JURECA [71] at Jülich Supercomputing Centre (JSC) as well as the Gauss Centre for Supercomputing e.V. [72] for providing computing time on the GCS Supercomputer SuperMUCNG at Leibniz Supercomputing Centre [73]. GCS is the alliance of the three national supercomputing centers HLRS (Universität Stuttgart), JSC (Forschungszentrum Jülich), and LRZ (Bayerische Akademie der Wissenschaften), funded by the German Federal Ministry of Education and Research (BMBF) and the German State Ministries for Research of Baden-Württemberg (MWK), Bayern (StMWFK) and Nordrhein-Westfalen (MIWF). Computer time on the DFG-funded Ara cluster at the Friedrich Schiller University Jena is acknowledged. Additional simulations were carried out on the Regensburg Athene2 cluster and the SFB/TRR 55 QPACE 3 machine.
[1] V. D. Burkert, Annu. Rev. Nucl. Part. Sci. 68, 405 (2018).

[2] R. Abdul Khalek et al., arXiv:2103.05419.

[3] J. W. Qiu and G. F. Sterman, Phys. Rev. Lett. 67, 2264 (1991).

[4] D. W. Sivers, Phys. Rev. D 41, 83 (1990).

[5] I. Scimemi, A. Tarasov, and A. Vladimirov, J. High Energy Phys. 05 (2019) 125.

[6] M. Bury, A. Prokudin, and A. Vladimirov, Phys. Rev. Lett. 126, 112002 (2021).

[7] S. Wandzura and F. Wilczek, Phys. Lett. 72B, 195 (1977).

[8] R. L. Jaffe and X. D. Ji, Phys. Rev. D 43, 724 (1991).

[9] M. Burkardt, Phys. Rev. D 88, 114502 (2013).

[10] F. P. Aslan, M. Burkardt, and M. Schlegel, Phys. Rev. D 100, 096021 (2019).

[11] M. Göckeler, R. Horsley, D. Pleiter, P. E. L. Rakow, A. Schäfer, G. Schierholz, H. Stüben, and J. M. Zanotti, Phys. Rev. D 72, 054507 (2005).

[12] K. Abe et al. (E154 Collaboration), Phys. Lett. B 404, 377 (1997).

[13] K. Abe et al. (E143 Collaboration), Phys. Rev. D 58, 112003 (1998).

[14] P. L. Anthony et al. (E155 Collaboration), Phys. Lett. B 553, 18 (2003).

[15] X. Zheng et al. (Jefferson Lab Hall A Collaboration), Phys. Rev. C 70, 065207 (2004).

[16] A. Airapetian et al. (HERMES Collaboration), Eur. Phys. J. C 72, 1921 (2012).
[17] M. Posik et al. (Jefferson Lab Hall A Collaboration), Phys. Rev. Lett. 113, 022002 (2014).

[18] D. Flay et al. (Jefferson Lab Hall A Collaboration), Phys. Rev. D 94, 052003 (2016).

[19] W. Armstrong et al. (SANE Collaboration), Phys. Rev. Lett. 122, 022002 (2019).

[20] V. M. Braun, Y. Ji, and A. Vladimirov, J. High Energy Phys. 05 (2021) 086.

[21] X. Ji, Phys. Rev. Lett. 110, 262002 (2013).

[22] K. Cichy and M. Constantinou, Adv. High Energy Phys. 2019, 3036904 (2019).

[23] X. Ji, Y. S. Liu, Y. Liu, J. H. Zhang, and Y. Zhao, Rev. Mod. Phys. 93, 035005 (2021).

[24] X. Ji, Y. Liu, A. Schäfer, W. Wang, Y. B. Yang, J. H. Zhang, and Y. Zhao, Nucl. Phys. B964, 115311 (2021).

[25] Y. K. Huo et al. (Lattice Parton Collaboration), Nucl. Phys. B969, 115443 (2021).

[26] C. Alexandrou, K. Cichy, M. Constantinou, J. R. Green, K. Hadjiyiannakou, K. Jansen, F. Manigrasso, A. Scapellato, and F. Steffens, Phys. Rev. D 103, 094512 (2021).

[27] M. Constantinou, Eur. Phys. J. A 57, 77 (2021).

[28] R. L. Jaffe, Comments Nucl. Part. Phys. 19, 239 (1990).

[29] E. B. Zijlstra and W. L. van Neerven, Nucl. Phys. B417, 61 (1994); B426, 245(E) (1994); B501, 599(E) (1997); B773, 105(E) (2007).

[30] B. Ehrnsperger, A. Schäfer, and L. Mankiewicz, Phys. Lett. B 323, 439 (1994). 
[31] E. V. Shuryak and A. I. Vainshtein, Nucl. Phys. B201, 141 (1982).

[32] A. P. Bukhvostov, E. A. Kuraev, and L. N. Lipatov, JETP Lett. 37, 482 (1983).

[33] A. P. Bukhvostov, E. A. Kuraev, and L. N. Lipatov, Sov. Phys. JETP 60, 22 (1984).

[34] P. G. Ratcliffe, Nucl. Phys. B264, 493 (1986).

[35] I. I. Balitsky and V. M. Braun, Nucl. Phys. B311, 541 (1989).

[36] X. D. Ji and C. H. Chou, Phys. Rev. D 42, 3637 (1990).

[37] J. Kodaira, Y. Yasui, and T. Uematsu, Phys. Lett. B 344, 348 (1995).

[38] J. Kodaira, Y. Yasui, K. Tanaka, and T. Uematsu, Phys. Lett. B 387, 855 (1996).

[39] M. Baake, B. Gemünden, and R. Oedingen, J. Math. Phys. (N.Y.) 23, 944 (1982); 23, 2595(E) (1982).

[40] J. E. Mandula, G. Zweig, and J. Govaerts, Nucl. Phys. B228, 109 (1983).

[41] M. Göckeler, R. Horsley, E. M. Ilgenfritz, H. Perlt, P. E. L. Rakow, G. Schierholz, and A. Schiller, Phys. Rev. D 54, 5705 (1996).

[42] G. S. Bali, S. Bürger, S. Collins, M. Göckeler, M. Gruber, S. Piemonte, A. Schäfer, A. Sternbeck, and P. Wein, Phys. Rev. D 103, 094511 (2021).

[43] R. Arthur and P. A. Boyle (RBC and UKQCD Collaborations), Phys. Rev. D 83, 114511 (2011).

[44] M. Bruno et al., J. High Energy Phys. 02 (2015) 043.

[45] M. Lüscher and S. Schaefer, Comput. Phys. Commun. 184, 519 (2013).

[46] S. Güsken, Nucl. Phys. B, Proc. Suppl. 17, 361 (1990).

[47] M. Falcioni, M. L. Paciello, G. Parisi, and B. Taglienti, Nucl. Phys. B251, 624 (1985).

[48] M. Lüscher and S. Schaefer, J. High Energy Phys. 07 (2011) 036.

[49] M. Bruno, T. Korzec, and S. Schaefer, Phys. Rev. D 95, 074504 (2017).

[50] G. S. Bali, E. E. Scholz, J. Simeth, and W. Söldner (RQCD Collaboration), Phys. Rev. D 94, 074501 (2016).

[51] R. Babich, J. Brannick, R. C. Brower, M. A. Clark, T. A. Manteuffel, S. F. McCormick, J. C. Osborn, and C. Rebbi, Phys. Rev. Lett. 105, 201602 (2010).

[52] A. Frommer, K. Kahl, S. Krieg, B. Leder, and M. Rottmann, SIAM J. Sci. Comput. 36, A1581 (2014).
[53] S. Heybrock, M. Rottmann, P. Georg, and T. Wettig, Proc. Sci., LATTICE2015 (2016) 036 [arXiv:1512.04506].

[54] D. Richtmann, S. Heybrock, and T. Wettig, Proc. Sci., LATTICE2015 (2016) 035 [arXiv:1601.03184].

[55] P. Georg, D. Richtmann, and T. Wettig, Proc. Sci., LATTICE2016 (2017) 361 [arXiv:1701.08521].

[56] P. Georg, D. Richtmann, and T. Wettig, EPJ Web Conf. 175, 02007 (2018).

[57] M. Lüscher, J. High Energy Phys. 07 (2007) 081.

[58] M. Lüscher, J. High Energy Phys. 12 (2007) 011.

[59] G. Martinelli and C. T. Sachrajda, Nucl. Phys. B316, 355 (1989).

[60] J. D. Bratt et al. (LHPC Collaboration), Phys. Rev. D 82, 094502 (2010).

[61] R. G. Edwards and B. Joó (SciDAC, LHPC and UKQCD Collaborations), Nucl. Phys. B, Proc. Suppl. 140, 832 (2005).

[62] M. Osipenko, S. Simula, W. Melnitchouk, P. E. Bosted, V. Burkert, E. Christy, K. Griffioen, C. Keppel, S. E. Kuhn, and G. Ricco, Phys. Rev. D 71, 054007 (2005).

[63] N. Sato, W. Melnitchouk, S. E. Kuhn, J. J. Ethier, and A. Accardi (JAM Collaboration), Phys. Rev. D 93, 074005 (2016).

[64] Z. Fan, X. Gao, R. Li, H. W. Lin, N. Karthik, S. Mukherjee, P. Petreczky, S. Syritsyn, Y. B. Yang, and R. Zhang, Phys. Rev. D 102, 074504 (2020).

[65] R. G. Edwards, G. Fleming, P. Hägler, J. W. Negele, K. Orginos, A. V. Pochinsky, D. B. Renner, D. G. Richards, and W. Schroers, Proc. Sci., LAT2006 (2006) 121 [arXiv: hep-lat/0610007].

[66] D. Dolgov et al. (LHPC and SESAM Collaborations), Phys. Rev. D 66, 034506 (2002).

[67] S. Bhattacharya, K. Cichy, M. Constantinou, A. Metz, A. Scapellato, and F. Steffens, Phys. Rev. D 102, 111501 (2020).

[68] http://wiki-zeuthen.desy.de/CLS/CLS.

[69] A. Nobile, Proc. Sci., LATTICE2010 (2010) 034 [arXiv: 1109.4279].

[70] M. Lüscher and S. Schaefer, http://cern.ch/luscher/openQCD (2012).

[71] Jülich Supercomputing Centre, J. Large-Scale Res. Facil. 4, A132 (2018).

[72] https://www.gauss-centre.eu.

[73] https://www.lrz.de. 